\title{
Bi-Lipschitz parameterization of surfaces
}

\author{
Mario Bonk • Urs Lang
}

Received: 23 September 2002 / Revised version: 12 October 2002 /

Published online: 16 May 2003 - @ Springer-Verlag 2003

\begin{abstract}
We consider surfaces $Z$ homeomorphic to the plane with complete, possibly singular Riemannian metrics. If we have $\int_{Z} K^{+}<2 \pi-\epsilon$ for the positive and $\int_{Z} K^{-}<C$ for the negative part of the integral curvature, then $Z$ is $L$-bi-Lipschitz equivalent to $\mathbb{R}^{2}$ with $L$ depending only on $\epsilon>0$ and $C>0$. This result implies a conjecture by J. Fu.
\end{abstract}

\section{Introduction}

Suppose $\left(X, d_{X}\right)$ and $\left(Y, d_{Y}\right)$ are metric spaces. A map $f: X \rightarrow Y$ is called bi-Lipschitz if there exists a constant $L \geq 1$ (a bi-Lipschitz constant for $f$ ) such that

$$
\frac{1}{L} d_{X}(x, y) \leq d_{Y}(f(x), f(y)) \leq L d_{X}(x, y) \quad \text { for } x, y \in X .
$$

If there exists a bi-Lipschitz homeomorphism between two metric spaces $X$ and $Y$ with bi-Lipschitz constant $L$, then the spaces are called L-bi-Lipschitz equivalent or just bi-Lipschitz equivalent, if the constant does not matter. It is an important problem to characterize the Euclidean spaces $\mathbb{R}^{n}$ up to bi-Lipschitz equivalence (see [Se3] for related information). This is easy for $n=1$, but already for $n=2$ this seems to be an exceedingly hard problem. In [To1,To2, MS] the question was studied when the graph of a function on $\mathbb{R}^{n}$ is bi-Lipschitz equivalent to $\mathbb{R}^{n}$ (see also [Se1]). For $n=2$ these results were strengthened by J. Fu. He considered complete smooth Riemannian surfaces $Z$ homeomorphic to the plane with Gaussian curvature $K$ and showed that if the absolute integral curvature of $Z$ satisfies $\int_{Z}|K| \leq \epsilon_{0}$, where $\epsilon_{0}$ is a (small) positive numerical constant, then $Z$ is bi-Lipschitz equivalent to $\mathbb{R}^{2}$ [Fu, Theorem D].

M. BONK*

Department of Mathematics, University of Michigan, Ann Arbor, MI 48109-1109, USA (e-mail: mbonk@umich.edu)

U. LANG

Departement Mathematik, ETH Zentrum, CH-8092 Zürich, Switzerland

(e-mail: lang@math.ethz.ch)

*Supported by NSF grant DMS-0200566. 
Fu conjectured that this statement remains true under the weaker assumption $\int_{Z} K^{+}<2 \pi$ and $\int_{Z} K^{-}<\infty$. In the present paper we establish Fu's conjecture by proving the following more general theorem.

Theorem 1.1. Suppose $Z$ is a complete Aleksandrov surface homeomorphic to the plane. If $\int_{Z} K^{+}<2 \pi$ and $\int_{Z} K^{-}<\infty$, then $Z$ is L-bi-Lipschitz equivalent to $\mathbb{R}^{2}$ with

$$
L=\left(\frac{2 \pi+\int_{Z} K^{-}}{2 \pi-\int_{Z} K^{+}}\right)^{1 / 2} .
$$

Here an Aleksandrov surface is a singular surface with locally bounded total variation of the integral curvature (see below for a precise definition). The class of Aleksandrov surfaces contains all smooth Riemannian surfaces and all polyhedral surfaces, for example. Roughly speaking, Aleksandrov surfaces form the largest class of surfaces on which a notion of integral curvature can be defined as a signed Borel measure $\mu$ on the surface. In the smooth case the integral curvature $\mu(E)$ of a Borel subset $E$ is just the integral $\int_{E} K$ of the pointwise defined Gaussian curvature $K$ over the set $E$. Here integration is with respect to the Riemannian area on the surface. In the statement of Theorem 1.1, $\int_{Z} K^{+}$and $\int_{Z} K^{-}$stand for $\mu^{+}(Z)$ and $\mu^{-}(Z)$, respectively, where $\mu=\mu^{+}-\mu^{-}$is the Jordan decomposition of $\mu$.

Even if we only want to guarantee that the surface $Z$ in Theorem 1.1 is bi-Lipschitz equivalent to $\mathbb{R}^{2}$ without precise control of the bi-Lipschitz constant, then the bound $2 \pi$ for the positive integral curvature is best possible. To see this let $Z$ be a one-sided infinite cylinder whose finite end is closed off by a hemisphere. To be specific, let

$$
Z=\left\{(x, y, z) \in \mathbb{R}^{3}: x^{2}+y^{2}=1, z>0 \text { or } x^{2}+y^{2}+z^{2}=1, z \leq 0\right\} .
$$

Then $Z$ is an Aleksandrov surface with $\mu^{+}(Z)=2 \pi$ and $\mu^{-}(Z)=0$. On the other hand, $Z$ is not bi-Lipschitz equivalent to $\mathbb{R}^{2}$. For if this were the case, then the area of a disk of radius $R$ (in the intrinsic metric) on $Z$ would grow as $R^{2}$ for $R \rightarrow \infty$. But this is obviously not true for the disks on $Z$.

Theorem 1.1 can be formulated in the following weaker form: Suppose $Z$ is a complete Aleksandrov surface homeomorphic to the plane. If $\mu^{+}(Z) \leq 2 \pi-\epsilon$ and $\mu^{-}(Z) \leq C$, then $Z$ is L-bi-Lipschitz equivalent to $\mathbb{R}^{2}$ with $L$ depending only on $\epsilon>0$ and $C>0$. The dependence of the bi-Lipschitz constant on $\epsilon$ and $C$ is unavoidable in this statement. This can be seen by considering the Euclidean cone $C_{\lambda}$ over a circle of length $\lambda>0$. Its integral curvature is a Dirac measure of total mass $2 \pi-\lambda$ located at the vertex of $C_{\lambda}$. The cone $C_{\lambda}$ is $L(\lambda)$-bi-Lipschitz equivalent to $\mathbb{R}^{2}$, but we necessarily have $L(\lambda) \rightarrow \infty$ as $\lambda \rightarrow 0$ or $\lambda \rightarrow \infty$. To see this, assume first that $0<\lambda \leq 2 \pi$ and let $\Gamma$ be the unit circle in $C_{\lambda}$ centered at the vertex of $C_{\lambda}$. The curve $\Gamma$ has length $\lambda$. Under an $L$-bi-Lipschitz equivalence onto $\mathbb{R}^{2}$, the curve $\Gamma$ is mapped onto a Jordan curve $\Gamma^{\prime}$ which contains a 
Euclidean disk of radius $1 / L$ in its interior region. Hence the length of $\Gamma^{\prime}$ is at least $2 \pi / L$. On the other hand, the length of $\Gamma^{\prime}$ is bounded by $\lambda L$. This shows $L \geq(2 \pi / \lambda)^{1 / 2} \rightarrow \infty$ as $\lambda \rightarrow 0$. Similarly, let $\lambda \geq 2 \pi$ and assume that we have an $L$-bi-Lipschitz equivalence of $C_{\lambda}$ onto $\mathbb{R}^{2}$. Then the vertex $o$ of $C_{\lambda}$ is mapped to a point $p_{0} \in \mathbb{R}^{2}$. The unit circle $\Gamma^{\prime}$ in $\mathbb{R}^{2}$ centered at $p_{0}$ is the image of a Jordan curve $\Gamma$ containing a disk of radius $1 / L$ centered at $o$ in its interior. Hence the length of $\Gamma$ is at least $\lambda / L$. On the other hand, this length is bounded by $2 \pi L$. This shows $L \geq(\lambda /(2 \pi))^{1 / 2} \rightarrow \infty$ as $\lambda \rightarrow \infty$.

In view of these examples the bi-Lipschitz constant $L$ given in Theorem 1.1 is optimal (for given values of $\int_{Z} K^{+}$or $\int_{Z} K^{-}$) if the surface $Z$ is non-negatively or non-positively curved in addition. It seems that this is not true in the general case. For related results on optimal bi-Lipschitz constants see [Mi].

The proof of Theorem 1.1 can be outlined as follows. By an approximation argument the general case of Aleksandrov surfaces can be reduced to the case of polyhedral surfaces, that is, surfaces which are everywhere flat except at finitely many vertices, where the surface locally looks like the neighborhood of the vertex in a cone $C_{\lambda}$. The idea is now to find pairwise disjoint flat sectors on the polyhedral surface $X$ whose set of vertices agrees with the set of vertices of the surface. In general, there will be several sectors for each vertex on the surface. Using a bi-Lipschitz deformation of these sectors, the total angle at each vertex is adjusted, so that it becomes $2 \pi$. The new surface obtained in this way is flat everywhere. Hence it must be isometric to $\mathbb{R}^{2}$ and we get the desired bi-Lipschitz equivalence of $X$ and $\mathbb{R}^{2}$. The main difficulty lies in the choice of the sectors, in particular, if one wants to keep precise control over the bi-Lipschitz constant. As a model case for this type of argument we included Lemma 5.1. It is actually not needed for the proof, but it serves to illustrate the main point.

The paper is organized as follows. In Section 2 we set up notation, formulate basic definitions and cite facts from the theory of Aleksandrov surfaces. In Section 3 we show that a complete Aleksandrov surface $Z$ which is homeomorphic to the plane and satisfies $\mu^{+}(Z)<2 \pi$ admits exhaustions by convex polygons. This result is later needed for the approximation argument. In Section 4 we fix some terminology related to polyhedral surfaces. Proposition 5.2 in Section 5 is one of the core results for the proof of Theorem 1.1. Here we construct the sectors mentioned above if the surface is a polyhedral halfplane. To apply this result to polyhedral planes we have to consider splittings of such surfaces into appropriate polyhedral halfplanes. This is discussed in Section 6. The desired splittings are rather easy to construct in the smooth case (cf. Proposition 6.1), but additional complications arise in the polyhedral case. These are overcome in Proposition 6.2 and we obtain the polyhedral plane version (cf. Proposition 6.3) of Proposition 5.2. We note that the considerations in Section 6 could have been somewhat simplified at the cost of a worse bi-Lipschitz constant in our main theorem. The second core result for the proof of Theorem 1.1 is Theorem 7.1 in Section 7. This statement 
may be of independent interest as it provides a polyhedral halfplane version of our main result. Its proof illustrates the technique of curvature elimination by biLipschitz deformations of the sectors that were found in Section 5. This theorem and the splitting results of Section 6 easily lead to the polyhedral version of our main result (Theorem 7.2). It remains to run an approximation argument to get a proof in the general case. This is done in Section 8. The final Section 9 is for concluding remarks.

At first reading the reader is advised to skim through Section 4 for notation and terminology for polyhedral surfaces and then proceed to the core arguments provided in the proofs of Proposition 5.2 and Theorem 7.1. Lemma 5.1 will provide some guidance for understanding the technicalities in the proof of Proposition 5.2.

\section{Aleksandrov surfaces}

In this section we set up notation and briefly review some facts from the theory of Aleksandrov surfaces or surfaces of bounded curvature as they were called by A.D. Aleksandrov and his school. See [Re] for an extended survey and [AZ] for a detailed account. This class should not be confused with the class of surfaces (or more general spaces) with curvature bounded above or below which were also studied by Aleksandrov.

Suppose $X$ is a metric space with metric $d=d_{X}$. Let $I \subset \mathbb{R}$ be an interval. We denote the length of a rectifiable curve $\gamma: I \rightarrow X$ by $L(\gamma)$. We assume the metric $d$ to be intrinsic, i.e., the distance of any two points equals the infimum of the lengths of curves connecting these points. If $M$ is a subset of $X$ with the property that any two points in $M$ can be connected by some rectifiable curve in $M$, then the induced intrinsic metric $d_{M}$ on $M$ is defined by

$$
d_{M}(x, y)=\inf _{\gamma} L(\gamma) \quad \text { for } x, y \in M,
$$

where the infimum is taken over all rectifiable curves in $M$ with endpoints $x$ and $y$. We denote the distance between two sets $M, N \subset X$ by

$$
\operatorname{dist}(M, N)=\inf \{d(x, y): x \in M, y \in N\} .
$$

The open ball and the closed ball of radius $r>0$ centered at $x \in X$ are denoted $B(x, r)$ and $\bar{B}(x, r)$, respectively. A metric space is proper if its closed balls are compact. If $M \subset X$ is a set, then $\operatorname{cl}(M)$ is the closure and $\operatorname{int}(M)$ the interior of $M$. For a function $h: X \rightarrow \mathbb{R}, \operatorname{spt}(h)=\operatorname{cl}(\{x \in X: h(x) \neq 0\})$ denotes its support.

A curve $\gamma: I \rightarrow X$ defined on a compact interval $I=[a, b]$ is called a minimizing geodesic segment if $L(\gamma)=d(\gamma(a), \gamma(b))$ and if $\gamma$ is parameterized with constant speed. The image $\operatorname{im}(\gamma)$ of a minimizing geodesic segment $\gamma$ with endpoints $x$ and $y$ is a simple (possibly degenerate) arc. We denote any such arc 
by $[x, y]$ and refer to it as a shortest arc. The notation is ambiguous as $[x, y]$ need not be uniquely determined by its endpoints. A metric space is called geodesic if every two points can be connected by a shortest arc. Every complete and locally compact intrinsic metric space is geodesic and proper. A subset $M \subset X$ is called convex if for all points $x, y \in M$, there exists a shortest arc $[x, y]$ contained in $M$. In this case the induced intrinsic metric $d_{M}$ agrees with the induced metric, i.e., the restriction of the ambient metric $d_{X}$ to $M$. Suppose $I \subset \mathbb{R}$ is a closed interval, and $\gamma: I \rightarrow X$ is a locally rectifiable curve in constant speed parameterization. If for every point $t \in I$ there exists a compact interval $J \subset I$ containing $t$ in its relative interior such that $\gamma \mid J$ is a minimizing geodesic segment, then $\gamma$ is called a geodesic. If $I$ is a compact interval, $I=[0, \infty)$, or $I=\mathbb{R}$, the geodesic will be called a geodesic segment, a geodesic ray, or a geodesic line. We use the same terms also for the respective images.

Suppose $\alpha$ and $\beta$ are simple arcs in $X$ with a common endpoint $o$. Let $x \in \alpha$, $y \in \beta, x, y \neq o$, and consider a Euclidean triangle with sides of lengths $d(o, x)$, $d(o, y), d(x, y)$. Let $\gamma(x, y) \in[0, \pi]$ be the angle opposite the side whose length is $d(x, y)$. The upper angle between $\alpha$ and $\beta$ is defined as

$$
\bar{L}_{o}(\alpha, \beta)=\limsup _{x, y \rightarrow o} \gamma(x, y) \in[0, \pi] .
$$

Here the points $x \in \alpha$ and $y \in \beta$ tend to $o$ along $\alpha$ and $\beta$, respectively.

A surface is a connected topological 2-manifold with (possibly empty) boundary. A metric $d$ on a surface is a metric whose induced topology agrees with the topology of the surface. In the following $Z$ stands for a surface without boundary with an intrinsic metric $d=d_{Z}$. Since it is enough for our purpose and since it simplifies some of the more technical aspects of the theory of Aleksandrov surfaces, we will in addition assume that $Z$ is homeomorphic to the plane and that $(Z, d)$ is a complete metric space.

A polygonal line in $Z$ is a curve of the form $\left[x_{1}, x_{2}\right] \cup\left[x_{2}, x_{3}\right] \cup \cdots \cup\left[x_{n-1}, x_{n}\right]$. A simple arc or a Jordan curve $J$ is a polygonal line, if and only if every point in $J$ has a neighborhood in $J$ consisting of one or two shortest arcs. A closed Jordan region is a compact subset of $Z$ homeomorphic to a closed disk. A Jordan polygon is a closed Jordan region $P$ whose boundary $\partial P$ is a polygonal line. If the closed Jordan region $P$ has rectifiable boundary $\partial P$, then it is called convex relative to its boundary if no arc $\alpha$ which lies outside $P$ except its endpoints $x, y \in \partial P$ is shorter than the corresponding arc $\beta$ on $\partial P$ with the same endpoints. Here the arc $\beta$ corresponding to $\alpha$ is the unique subarc of $\partial P$ with endpoints $x$ and $y$ such that $\beta$ is contained in the closed Jordan region bounded by $\alpha \cup(\partial P \backslash \beta)$. If $P$ is a Jordan polygon which is convex relative to its boundary, then it is also convex.

A simple triangle $\Delta$ is a Jordan polygon which is convex relative to its boundary and whose boundary can be written as a union of three distinguished non-overlapping non-degenerate shortest $\operatorname{arcs} \alpha_{1}=\left[x_{2}, x_{3}\right], \alpha_{2}=\left[x_{3}, x_{1}\right], \alpha_{3}=\left[x_{1}, x_{2}\right]$. Here we say that two subsets of a metric space are non-overlapping, if they do not 
have interior points in common. Note that the $\operatorname{arcs} \alpha_{i}$ are considered as subsets of the space $\partial \Delta$. The points $x_{i}$ are the vertices, the arcs $\alpha_{i}$ the edges of the triangle $\Delta$. The upper excess $\bar{\delta}(\Delta)$ of the simple triangle $\Delta$ is defined as

$$
\bar{\delta}(\Delta)=\bar{L}_{x_{1}}\left(\alpha_{2}, \alpha_{3}\right)+\bar{L}_{x_{2}}\left(\alpha_{3}, \alpha_{1}\right)+\bar{L}_{x_{3}}\left(\alpha_{1}, \alpha_{2}\right)-\pi .
$$

A surface $Z$ with intrinsic metric is called an Aleksandrov surface if for every point in $Z$ there exists a neighborhood $U$, homeomorphic to an open disk, and a constant $C(U)<\infty$ such that for any system $T_{1}, \ldots, T_{n}$ of non-overlapping simple triangles contained in $U$ we have the inequality

$$
\sum_{i=1}^{n} \bar{\delta}\left(T_{i}\right) \leq C(U) .
$$

Starting from this geometric definition one can build up a theory of surfaces with features that correspond to those from the differential geometry of smooth surfaces. In particular, one can define a notion of integral curvature as a signed measure $\mu_{Z}$ on an Aleksandrov surface $Z$. The subscript $Z$ will be dropped, if the surface is understood.

According to work by Reshetnyak (see [Re, Section 7]) and A. Huber [Hu1], the geometric definition of Aleksandrov surfaces is equivalent to the following analytic definition. A topological surface $Z$ with an intrinsic metric is an Aleksandrov surface if and only if there exists an atlas of complex charts on $Z$ such that the metric can locally be defined by a length element of the form

$$
e^{u(z)}|d z|,
$$

where $z$ is a complex local coordinate and $u$ is a difference of two subharmonic functions such that $\exp u$ is locally integrable on analytic curves in the $z$-plane. The generalized Laplacian $-\Delta u$ is a signed Borel measure $\mu$ on the surface, which represents the integral curvature. The density $\exp u$ is locally integrable on analytic curves if and only if every atom of $\mu$ has mass less than $2 \pi$. In this paper we will not adopt this analytic point of view, but we will work in a geometric framework. See Section 9 for additional discussion.

We will utilize a version of the Gauss-Bonnet theorem on our Aleksandrov surface $Z$. If $P \subset Z$ is a Jordan polygon, then one can associate a notion of turn or rotation of the boundary considered as a measure on $\partial P$. We have two signed Borel measures $\tau_{\text {in }}$ and $\tau_{\text {out }}$ (the "inner" and the "outer" turn) which describe the rotation of Borel subsets of $\partial P$ depending on whether we consider the rotation from inside or outside the Jordan region $P$ (see [AZ, Chapters VI and IX] for a discussion and precise definitions). In the smooth Riemannian case, $\tau_{\text {in }}$ corresponds to the geodesic curvature of subsets of $\partial P$ and we have $\tau_{\text {out }}=-\tau_{\text {in }}$. For general Aleksandrov surfaces, we have the equality

$$
\tau_{\text {in }}(E)+\tau_{\text {out }}(E)=\mu(E) \text { for all Borel subsets } E \subset \partial P .
$$


A version of the Gauss-Bonnet theorem [AZ, Chapter VI, Theorem 5] can be stated as

$$
\mu(\operatorname{int}(P))+\tau_{\text {in }}(\partial P)=2 \pi .
$$

It is useful to have a more explicit representation of the measures $\tau_{\text {in }}$ and $\tau_{\text {out }}$. For this purpose we represent $\partial P$ as a union

$$
\partial P=\gamma_{1} \cup \gamma_{2} \cup \cdots \cup \gamma_{n},
$$

where $\gamma_{i}=\left[x_{i-1}, x_{i}\right]$ are non-overlapping shortest arcs with $\gamma_{i} \cap \gamma_{i+1}=\left\{x_{i}\right\}$ for $i=1, \ldots, n$ (here $\gamma_{n+1}=\gamma_{1}$ and $x_{n}=x_{0}$ ). The points $x_{i}$ are called the vertices of this representation of $\partial P$. The measures $\tau_{\text {in }}$ and $\tau_{\text {out }}$ can be decomposed into a sum of measures $\tau_{i}$ and $\tau_{i}^{\prime}$ supported on the interior of the shortest arc $\gamma_{i}$ and atoms supported in the vertices $x_{i}$. The measures $\tau_{i}$ and $\tau_{i}^{\prime}$ correspond to the turn of subsets of interior points of $\gamma_{i}$ as considered from inside or outside $P$. The total turn of every shortest arc is non-positive; this is independent of whether we consider the turn from the "left" or "right" (see [AZ, Chapter VI, Theorem 6]). Since a measure on an open interval is non-positive if and only if the measure of every subinterval is non-positive, we conclude $\tau_{i} \leq 0$ and $\tau_{i}^{\prime} \leq 0$. The subarcs $\gamma_{i}$ and $\gamma_{i+1}$ of $\partial P$ which have the common endpoint $x_{i}$ decompose each sufficiently small neighborhood of $x_{i}$ in $Z$ which is homeomorphic to a disk into two complementary closed sectors $S_{i}$ and $S_{i}^{\prime}$ with vertex $x_{i}$. One of these sectors, say $S_{i}$, will be contained in $P$ and the other one, $S_{i}^{\prime}$, in $Z \backslash \operatorname{int}(P)$. To a sector one can associate a sector angle (see [AZ, Chapter II, Section 5 and Chapter IV, Section 4] for a discussion of sectors and for the precise definition of sector angles). Let $\lambda_{i}$ and $\lambda_{i}^{\prime}$ be the angles of the sectors $S_{i}$ and $S_{i}^{\prime}$, respectively. If we denote by $\delta_{z}$ the Dirac measure of mass 1 at the point $z$, then we have (this is essentially [AZ, Chapter VI, Theorem 4])

$$
\tau_{\text {in }}=\sum_{i=1}^{n} \tau_{i}+\sum_{i=1}^{n}\left(\pi-\lambda_{i}\right) \delta_{x_{i}}
$$

and

$$
\tau_{\text {out }}=\sum_{i=1}^{n} \tau_{i}^{\prime}+\sum_{i=1}^{n}\left(\pi-\lambda_{i}^{\prime}\right) \delta_{x_{i}} .
$$

If the Jordan polygon $P$ is convex relative to its boundary, then we have $\lambda_{i}^{\prime} \geq \pi$ ([AZ, pp. 120-121, Theorem 6]). It follows that $\tau_{\text {out }} \leq 0$.

\section{Convex exhaustions of Aleksandrov surfaces}

Let $Z$ be a complete Aleksandrov surface homeomorphic to the plane. A curve $\gamma:[a, b] \rightarrow Z$ is called a loop if $\gamma(a)=\gamma(b)$. Fix an orientation of $Z$ and let $Q \subset Z$ be a closed Jordan region. We say that a loop $\gamma:[0,1] \rightarrow Z$ surrounds 
$Q$ if $\operatorname{im}(\gamma) \cap \operatorname{int}(Q)=\emptyset$ and if the winding number of $\gamma$ with respect to the points in $\operatorname{int}(Q)$ is non-zero. Note that the winding number of $\gamma$ with respect to a point in $\operatorname{int}(Q)$ is independent of the point. The property that loops surround a fixed Jordan region $Q$ is stable under limits and under small perturbations of loops in $Z \backslash \operatorname{int}(Q)$ ). If $J$ is a Jordan curve (given in some parameterization), then $J$ surrounds $Q$ if and only if $\operatorname{int}(Q)$ is contained in the Jordan region determined by $J$.

Lemma 3.1. Suppose $Z$ is a complete Aleksandrov surface homeomorphic to the plane. If $\mu^{+}(Z)<2 \pi$, then for every closed Jordan region $Q \subset Z$ there exists a Jordan curve $J^{\prime}$ that surrounds $Q$ and has minimal length among all loops surrounding $Q$.

The basic idea of the proof of the lemma goes back to Cohn-Vossen [CV].

Proof. For $n \in \mathbb{N}$ consider the class $C_{n}$ of all rectifiable loops $\gamma:[0,1] \rightarrow Z$ with $\operatorname{dist}(Q, \operatorname{im}(\gamma)) \leq n$ that surround $Q$. Since $\gamma(0)=\gamma(1)$ we can consider $\gamma$ as a 1-periodic function on $\mathbb{R}$. Among the loops in $C_{n}$ there exists a loop $\gamma_{n}$ of minimal length. We want to show that $\operatorname{im}\left(\gamma_{n}\right) \cap Q \neq \emptyset$ for $n \in \mathbb{N}$.

Suppose that we have $\operatorname{im}\left(\gamma_{n}\right) \cap Q=\emptyset$ for some $n \in \mathbb{N}$. Set $\gamma:=\gamma_{n}$ for simplicity, and assume that $\gamma$ is given in constant speed parameterization. We now consider two cases:

Case I. There is no interval $[a, b] \subset \mathbb{R}$ with $b-a<1$ such that $\gamma \mid[a, b]$ is a loop surrounding $Q$. Then $J=\operatorname{im}(\gamma)$ is a Jordan curve. For otherwise, there would exist numbers $c$ and $d$ such that $0 \leq c<d<1$ with $\gamma(c)=\gamma(d)$. By the additivity of winding numbers under compositions of loops, it follows that one of the loops $\gamma \mid[c, d]$ or $\gamma \mid[d, c+1]$ surrounds $Q$, which is impossible by the assumption in this case.

There exist points $x_{0} \in J$ and $p_{0} \in Q$ such that $d\left(x_{0}, p_{0}\right)=\operatorname{dist}(J, Q) \leq n$. We may assume $\gamma(0)=\gamma(1)=x_{0}$. If $x \in J$ is a point with $x \neq x_{0}$, then each sufficiently small subarc of $J$ containing $x$ is a shortest arc. Otherwise, we could replace this subarc by a shortest arc with the same endpoints and obtain a loop in $C_{n}$ shorter than $\gamma$. Similarly, every sufficiently small subarc of $J$ having $x_{0}$ as one of its endpoints is a shortest arc. It follows that $\gamma$ is a geodesic loop (i.e., a loop which is a geodesic segment) and the two sector angles determined by $J$ and the vertex $x_{0}$ exist. Let $\lambda$ be the sector angle corresponding to the sector $S$ at $x_{0}$ contained in the closed Jordan region $P$ with boundary $\partial P=J$. The Gauss-Bonnet formula shows

$$
\mu(\operatorname{int}(P))+\tau_{\text {in }}\left(J \backslash\left\{x_{0}\right\}\right)+(\pi-\lambda)=2 \pi .
$$

Since $\gamma$ is a geodesic loop, we have $\tau_{\text {in }}\left(J \backslash\left\{x_{0}\right\}\right) \leq 0$. Let $\left[p_{0}, x_{0}\right]$ be a shortest arc. By definition of $p_{0}$ and $x_{0}$, we have $\left[p_{0}, x_{0}\right] \cap J=\left\{x_{0}\right\}$, and so travelling from $p_{0}$ along $\left[p_{0}, x_{0}\right]$, we approach $x_{0}$ through the sector $S$. If we had $\lambda<\pi$, 
then we could replace a small arc $\beta$ of $J$ containing $x_{0}$ in its interior by a shortest arc $\beta^{\prime}$ cutting across the sector $S$ with the same endpoints as $\beta$, but of strictly smaller length (see [BE, p. 67, Proposition 2] for more details). Then $\beta^{\prime}$ would meet $\left[p_{0}, x_{0}\right]$ and so $\operatorname{dist}\left(\beta^{\prime}, Q\right) \leq n$. In this way we could obtain a loop consisting of $\beta^{\prime}$ and $J \backslash \beta$ which surrounds $Q$ and is shorter than $\gamma$. Since this is impossible we conclude $\lambda \geq \pi$. By using (2) we arrive at

$$
\mu^{+}(Z) \geq \mu^{+}(\operatorname{int}(P))=\pi+\lambda-\tau_{\text {in }}\left(J \backslash\left\{x_{0}\right\}\right)+\mu^{-}(\operatorname{int}(P)) \geq 2 \pi .
$$

This contradicts our hypothesis $\mu^{+}(Z)<2 \pi$.

Case II. There is an interval $I=[a, b] \subset \mathbb{R}$ with $b-a<1$ such that $\gamma \mid[a, b]$ is a loop surrounding $Q$. Using Zorn's lemma one may assume that $[a, b]$ is minimal among such intervals. We claim that $\gamma \mid[a, b)$ is injective, i.e., $J=\gamma([a, b])$ is a Jordan curve. For otherwise, there would exist numbers $c$ and $d$ such that $a \leq c<d<b$ with $\gamma(c)=\gamma(d)$. Since $\gamma \mid[c, d]$ is a loop and $[c, d]$ is strictly contained in $I$, this loop cannot surround $Q$ by definition of $I$. By the additivity of winding numbers under compositions of loops, it follows that the loop $\gamma \mid[d, c+1]$ surrounds $Q$. But then $\operatorname{dist}(Q, \gamma([d, c+1]))>n$, for otherwise $\gamma \|[d, c+1]$ is a loop in $C_{n}$ which is shorter than $\gamma$. Since $\operatorname{dist}(Q, \operatorname{im}(\gamma)) \leq n$, this implies

$$
\operatorname{dist}(Q, \gamma([a, b])) \leq \operatorname{dist}(Q, \gamma([c, d])) \leq n
$$

and so $\gamma \mid[a, b] \in C_{n}$. But again this is impossible, since $\gamma \mid[a, b]$ is shorter than $\gamma$.

Let $Q^{\prime}:=\{z \in Z: \operatorname{dist}(z, Q) \leq n\}$. Since $\gamma \mid[a, b]$ is a loop surrounding $Q$ and $\gamma$ has minimal length among the loops in $C_{n}$, we must have $\operatorname{dist}\left(Q^{\prime}, J\right)>0$. Hence $Q^{\prime}$ lies in the open Jordan region $\Omega$ bounded by $J$. Moreover, there exists a point $t \in(b, a+1)$ such that $\gamma(t) \in Q^{\prime}$. Since $Q^{\prime}$ is compact, there exist points $p_{0} \in Q^{\prime}$ and $x_{0} \in J$ such that $d\left(p_{0}, x_{0}\right)=\operatorname{dist}\left(Q^{\prime}, J\right)>0$. Let $\left[p_{0}, x_{0}\right]$ be a shortest arc. By definition of $p_{0}$ and $x_{0}$ we have $\left[p_{0}, x_{0}\right] \cap Q^{\prime}=\left\{p_{0}\right\}$ and $\left[p_{0}, x_{0}\right] \cap J=\left\{x_{0}\right\}$. In particular, $\left[p_{0}, x_{0}\right] \cap \operatorname{int}(Q)=\emptyset$. This implies that we obtain a loop $\tilde{\gamma}$ in $C_{n}$ if we start at $p_{0}$, follow the $\operatorname{arc}\left[p_{0}, x_{0}\right]$ up to the endpoint $x_{0}$, go around $J$ once, and then return to $p_{0}$ along $\left[p_{0}, x_{0}\right]$ travelling in opposite direction. Note that

$$
\begin{aligned}
L(\tilde{\gamma}) & =L(J)+2 L\left(\left[p_{0}, x_{0}\right]\right) \\
& \leq L(\gamma \mid[a, b])+L(\gamma \mid[b, t])+L(\gamma \mid[t, a+1]) \\
& =L(\gamma) .
\end{aligned}
$$

Since $\gamma$ has minimal length among the loops in $C_{n}$, and $\tilde{\gamma} \in C_{n}$, we must have equality in this inequality. In particular, $\tilde{\gamma}$ has also minimal length among the loops in $C_{n}$. Arguing as in the previous case, we see that if we consider $x_{0}$ as the initial point and the endpoint of $J$, then in arclength parameterization $J$ is a geodesic loop and the sector angle $\lambda$ of the sector $S \subset \operatorname{cl}(\Omega)$ at $x_{0}$ is well defined. 
Again $\left[p_{0}, x_{0}\right]$ approaches $x_{0}$ through the sector $S$ and using the fact that $\tilde{\gamma} \in C_{n}$ has minimal length, we see that $\lambda \geq \pi$. As in the previous case, this leads to a contradiction by the Gauss-Bonnet formula.

Both cases exhaust all possibilities and lead to a contradiction. This means that the initial assumption that $\operatorname{im}\left(\gamma_{n}\right) \cap Q=\emptyset$ for some $n \in \mathbb{N}$ is impossible. Therefore, $\gamma_{n} \cap Q \neq \emptyset$ for all $n \in \mathbb{N}$. Let $C_{\infty}$ be the class of all loops surrounding $Q$. Then $C_{\infty}=\bigcup_{n \in \mathbb{N}} C_{n}$ and so

$$
\inf _{\gamma \in C_{\infty}} L(\gamma)=\inf _{n \in \mathbb{N}} L\left(\gamma_{n}\right) .
$$

Since $\gamma_{n} \cap Q \neq \emptyset$ for all $n \in \mathbb{N}$, the loops $\gamma_{n}$ stay inside a compact subset of $Z$ and by extracting a sublimit of these loops we can find a loop $\gamma_{\infty} \in C_{\infty}$ with

$$
L\left(\gamma_{\infty}\right)=\inf _{\gamma \in C_{\infty}} L(\gamma) .
$$

We may assume that $\gamma_{\infty}:[0,1] \rightarrow Z$ is given in constant speed parameterization. Arguing as above, we see that $\gamma_{\infty} \mid[0,1)$ is injective, and so $J^{\prime}=\gamma_{\infty}([0,1])$ is a Jordan curve.

Lemma 3.2. Suppose $Z$ is a complete Aleksandrov surface homeomorphic to the plane. If $\mu^{+}(Z)<2 \pi$, then for every Jordan polygon $Q \subset Z$ there exists a Jordan polygon $P$ containing $Q$ which is convex relative to its boundary.

A related result for smooth Riemannian surfaces was proved in [Ba1, Proposition 2].

Proof. Let $J^{\prime}$ be the Jordan curve produced in the previous lemma. As in this lemma, we denote by $C_{\infty}$ the set of loops surrounding $Q$. We will replace $J^{\prime}$ by a Jordan curve $J^{\prime \prime}$ in $C_{\infty}$ which is also length minimizing among the loops surrounding $Q$ and has the additional property that $J^{\prime \prime}$ is a polygonal line. Due to the minimization property of $J^{\prime}$, each point in $J^{\prime} \backslash Q$ lies in the interior of a subarc of $J^{\prime}$ which is a shortest arc; so the difficulties are caused by the points in $J^{\prime} \cap Q$. To obtain $J^{\prime \prime}$ we will modify $J^{\prime}$ so that $J^{\prime \prime}$ intersects $Q$ in a controlled manner. Since $J^{\prime} \cap \operatorname{int}(Q)=\emptyset$, the curve $J^{\prime}$ can only intersect the boundary of $Q$.

Since $\partial Q$ is a polygonal line, we can represent the boundary of $Q$ as the union of non-overlapping shortest $\operatorname{arcs} \alpha_{1}, \ldots, \alpha_{k}$. Let $\alpha$ be one of these arcs. Suppose $\alpha \cap J^{\prime}$ consists of more than one point. Then there exists a smallest non-degenerate subarc $\alpha^{\prime} \subset \alpha$ containing $\alpha \cap J^{\prime}$. The endpoints $x$ and $y$ of $\alpha^{\prime}$ lie in $J^{\prime}$. We can replace one of the two subarcs of $J^{\prime}$ with endpoints $x$ and $y$ by $\alpha^{\prime}$ to obtain a new Jordan curve which still surrounds $Q$. Moreover, the length of this new curve is not longer than $J^{\prime}$, since $\alpha^{\prime}$ is a shortest arc as a subarc of a shortest arc. By successive replacements of this type, we obtain a Jordan curve $J^{\prime \prime}$ in $C_{\infty}$ which has minimal length among the loops in $C_{\infty}$ and has the the additional property 
that $J^{\prime \prime} \cap \alpha_{i}$ is connected for $i=1, \ldots, k$ (this is a particular instance of the technique of "removal of superfluous intersections"; see [AZ, p. 51] and [Re, p. 79]). It follows that $J^{\prime \prime} \cap \partial Q$ consists of finitely many disjoint connected components. If $J^{\prime \prime} \cap \partial Q=\partial Q$, then $J^{\prime \prime}=\partial Q$ and $J^{\prime \prime}$ is a polygonal line. Otherwise, the connected components of $J^{\prime \prime} \cap \partial Q$ are either single points or simple arcs which are polygonal lines and form subarcs of both $J^{\prime \prime}$ and $\partial Q$. Let $\beta$ be one of these components and $z$ an endpoint of $\beta$ (i.e., $z=\beta$ if $\beta$ is a point). Consider any small subarc $\gamma$ of $J^{\prime \prime}$ with endpoint $z$. We claim that if $x \in \gamma \backslash\{z\}$ is sufficiently close to $z$, then the subarc $\gamma(x, z)$ of $\gamma$ between $x$ and $z$ is a shortest arc. This statement is obviously true if $\gamma(x, z)$ is contained in the connected component of $J^{\prime \prime} \cap \partial Q$ which contains $z$. In the other case we have $\gamma(x, z) \cap \partial Q=\{z\}$ assuming $d(x, z)$ is small enough. If $\gamma(x, z)$ is not a shortest arc, then $d(x, z)<L(\gamma(x, z))$. Consider a shortest arc $[x, z]$. Since $x$ does not belong to $Q$, there is a first point $y \in[x, z] \cap Q$ as we travel along $[x, z]$ starting from $x$. Since $\partial Q$ is a polygonal line and $y \in \partial Q$, we may assume that $x$ was chosen so close to $z$ that there is a (possibly degenerate) shortest arc $[y, z] \subset \partial Q$. Note that it is not necessarily true that for this segment we have $[y, z] \subset[x, z]$, but in any case

$$
L([x, z])=L([x, y])+L([y, z]),
$$

where $[x, y] \subset[x, z]$. Using this we obtain

$$
L([x, y])+L([y, z])=d(x, z)<L(\gamma(x, z)) .
$$

If the $\operatorname{arc} \gamma(x, z)$ of $J^{\prime \prime}$ is replaced by $[x, y] \cup[y, z]$ we obtain a loop $\tilde{\gamma}$ which does not meet int $(Q)$. Since winding numbers are invariant under small perturbations of loops, $\tilde{\gamma}$ will lie in $C_{\infty}$, assuming that $x$ was chosen sufficiently close to $z$. This is impossible, since $\tilde{\gamma}$ is shorter than $J^{\prime \prime}$. This shows that $\gamma(x, z)$ is a shortest arc.

We conclude that there are finitely many shortest arcs on $J^{\prime \prime}$ covering an open neighborhood of $Q \cap J^{\prime \prime}$ in $J^{\prime \prime}$. On the other hand, the minimality property of $J^{\prime \prime}$ implies that every point in $J^{\prime \prime} \backslash Q$ is contained in the interior of a subarc of $J^{\prime \prime}$ which is a shortest arc. This and a simple covering argument implies that $J^{\prime \prime}$ is a polygonal line. The closure $P$ of the interior region of $J^{\prime \prime}$ is a Jordan polygon containing $Q$. Suppose $\alpha$ is an arc which lies outside $P$ except its endpoints $x, y \in \partial P$. If we replace the corresponding $\operatorname{arc} \beta$ of $J^{\prime \prime}$ by $\alpha$, then we obtain a Jordan curve surrounding $Q$. The minimization property of $J^{\prime \prime}$ implies $L(\beta) \leq L(\alpha)$. This shows that $P$ is convex relative to its boundary.

Lemma 3.3. Suppose $Z$ is a complete Aleksandrov surface homeomorphic to the plane. If $\mu^{+}(Z)<2 \pi$, then for $n \in \mathbb{N}$ there exist Jordan polygons $P_{n} \subset Z$ which are convex relative to their boundaries and form an exhaustion of $Z$, i.e., $\operatorname{int}\left(P_{n}\right) \subset P_{n+1}$ for $n \in \mathbb{N}$ and $\bigcup_{n=1}^{\infty} P_{n}=Z$.

Proof. Every compact subset $Q$ of $Z$ lies in a Jordan polygon. This can be proved by approximating a Jordan curve containing $Q$ in its interior region by a polygonal 
line and then "removing superfluous intersections" (see [AZ, p. 51, Lemma 3] for a related statement). The claim now follows from Lemma 3.2.

\section{Polyhedral surfaces}

For $\lambda>0$ we denote by $C_{\lambda}^{\prime}$ the geodesic metric space induced by the pseudometric space

$$
\begin{gathered}
\left([0, \infty) \times[0, \lambda], d^{\prime}\right), \\
d^{\prime}\left((r, \theta),\left(r^{\prime}, \theta^{\prime}\right)\right)^{2}=r^{2}+r^{\prime 2}-2 r r^{\prime} \cos \left(\min \left\{\pi,\left|\theta-\theta^{\prime}\right|\right\}\right),
\end{gathered}
$$

i.e., $C_{\lambda}^{\prime}$ is the Euclidean cone over $[0, \lambda]$, and $C_{\pi}^{\prime}$ is isometric to a closed Euclidean halfplane. Similarly, $C_{\lambda}$ denotes the Euclidean cone over a circle of length $\lambda$, i.e., $C_{\lambda}$ is induced by the pseudometric space

$$
\begin{gathered}
([0, \infty) \times[0, \lambda], d), \\
d\left((r, \theta),\left(r^{\prime}, \theta^{\prime}\right)\right)^{2}=r^{2}+r^{\prime 2}-2 r r^{\prime} \cos \left(\min \left\{\pi,\left|\theta-\theta^{\prime}\right|, \lambda-\left|\theta-\theta^{\prime}\right|\right\}\right),
\end{gathered}
$$

and $C_{2 \pi}$ is isometric to the Euclidean plane. For $\epsilon>0$ let $C_{\lambda, \epsilon}^{\prime}:=C_{\lambda}^{\prime} \cap \bar{B}(o, \epsilon)$ and $C_{\lambda, \epsilon}:=C_{\lambda} \cap \bar{B}(o, \epsilon)$, where $\bar{B}(o, \epsilon)$ is the closed ball of radius $\epsilon$ around the vertex $o$ of the cone. By a polyhedral surface $X$ we mean a complete geodesic metric space with the property that for every $x \in X$ there exist $\lambda(x), \epsilon(x)>0$ such that the closed ball $\bar{B}(x, \epsilon(x))$ is isometric to either $C_{\lambda(x), \epsilon(x)}$ or $C_{\lambda(x), \epsilon(x)}^{\prime}$ so that $x$ corresponds to the vertex of the cone. According to these two cases, $x$ belongs to the interior $\operatorname{int}(X)$ or to the boundary $\partial X$ of $X$. The uniquely determined number $\lambda(x)$ is the total angle or (length of the) link at $x$. A polyhedral surface homeomorphic to a plane, a closed halfplane, or a closed disk will be called a polyhedral plane, polyhedral halfplane, or polyhedral disk, respectively. The integral curvature $\mu$ of a polyhedral surface $X$ is the signed measure whose support $\operatorname{spt}(\mu)$ is a discrete subset of $X$ and which satisfies

$$
\mu(x)=\left\{\begin{array}{lll}
2 \pi-\lambda(x) & \text { if } & x \in \operatorname{int}(X), \\
0 & \text { if } \quad x \in \partial X .
\end{array}\right.
$$

Here and throughout the paper $\mu(x)$ stands for $\mu(\{x\})$. Similarly, the turn $\tau$ of $\partial X$ is the discretely supported signed measure on $X$ satisfying

$$
\tau(x)=\left\{\begin{array}{lll}
\pi-\lambda(x) & \text { if } & x \in \partial X, \\
0 & \text { if } & x \in \operatorname{int}(X) .
\end{array}\right.
$$

It is convenient to combine $\mu$ and $\tau$ to a single measure

$$
v:=\mu+\tau
$$


which we refer to as the total measure of $X$. For a polyhedral disk $X$ the GaussBonnet formula now simply reads

$$
\nu(X)=2 \pi .
$$

Points in the support $\operatorname{spt}(\nu)$ of $v=\mu+\tau$ are called (interior or boundary) vertices of the polyhedral surface $X$. Note that, if $X$ is a polyhedral plane and $X=P^{\prime} \cup P^{\prime \prime}$ for two polyhedral halfplanes with $P^{\prime} \cap P^{\prime \prime}=\partial P^{\prime}=\partial P^{\prime \prime}$, each equipped with its induced intrinsic metric, then the total measures of $P^{\prime}$ and $P^{\prime \prime}$ add up to the integral curvature of $X$, i.e., $v_{P^{\prime}}+v_{P^{\prime \prime}}=\mu$, where it is understood that $v_{P^{\prime}}=0$ on $X \backslash P^{\prime}$ and $v_{P^{\prime \prime}}=0$ on $X \backslash P^{\prime \prime}$.

Every polyhedral surface without boundary is an Aleksandrov surface as defined in Section 2. Every polyhedral surface $X$ with boundary $\partial X \neq \emptyset$ is isometric to a closed polyhedral region $P$ in some Aleksandrov surface $Z$; the integral curvature $\mu$ of $X$ agrees with the restriction of $\mu_{Z}$ to int $(P)$, and the turn $\tau$ of $\partial X$ agrees with the measure $\tau_{\text {in }}$ on $\partial P$ as a subset of $Z$.

\section{Choosing sectors}

As mentioned in the introduction, the proof of our main result in the polyhedral case relies on the choice of a suitable family of flat sectors on a given polyhedral halfplane or plane. This is the content of Propositions 5.2 and 6.3. The idea of the construction is best illustrated by the following lemma which is not needed in the sequel but which motivated the whole approach.

By a convex polyhedron $P \subset \mathbb{R}^{2}$ we mean the intersection of a finite number of closed halfplanes, including the case $P=\mathbb{R}^{2}$. Every such surface $P$, endowed with the induced metric, is a polyhedral surface as defined in the previous section, with integral curvature $\mu=0$. For a convex polyhedron $P \subset \mathbb{R}^{2}$ homeomorphic to a closed halfplane, the total boundary turn satisfies $0 \leq \tau(\partial P) \leq \pi$ and agrees with the sum of the bending angles of $\partial P$. A sector $\Sigma \subset \mathbb{R}^{2}$ is the non-empty intersection of two closed halfplanes whose union is distinct from $\mathbb{R}^{2}$; in case $\Sigma$ is a halfplane, it comes with the choice of a vertex $y \in \partial \Sigma$. The angle $\beta \in(0, \pi]$ of a sector $\Sigma \subset \mathbb{R}^{2}$ is read off between the two rays in $\partial \Sigma$ issuing from the vertex of $\Sigma$.

Lemma 5.1. Let $P \subset \mathbb{R}^{2}$ be either $\mathbb{R}^{2}$ or a convex polyhedron homeomorphic to a closed halfplane, and suppose $\alpha \geq 0$ is a measure on $P$ with finite support $\operatorname{spt}(\alpha)$ and

$$
0<\alpha(P) \leq \begin{cases}\pi-\tau(\partial P) & \text { if } P \neq \mathbb{R}^{2}, \\ 2 \pi & \text { if } P=\mathbb{R}^{2} .\end{cases}
$$

Then there exist finitely many sectors $\Sigma_{1}, \ldots, \Sigma_{k} \subset P$ with vertices $y_{1}, \ldots, y_{k} \in$ $\operatorname{spt}(\alpha)$ and angles $\beta_{1}, \ldots, \beta_{k}$, respectively, such that $\operatorname{int}\left(\Sigma_{i}\right) \cap \operatorname{int}\left(\Sigma_{j}\right)=\emptyset$ for $i \neq j$ and $\sum_{y_{i}=x} \beta_{i}=\alpha(x)$ for every $x \in \operatorname{spt}(\alpha)$. 
Proof. We first treat the case $P \neq \mathbb{R}^{2}$. Let $P_{1}:=P$ and $\alpha_{1}:=\alpha$. The sectors $\Sigma_{i} \subset P$ are selected by repeating the following procedure consecutively for $i=1,2, \ldots$ Given $P_{i}$ and $\alpha_{i}$ with $\operatorname{spt}\left(\alpha_{i}\right) \subset P_{i}$ and $0<\alpha_{i}\left(P_{i}\right) \leq \pi-\tau\left(\partial P_{i}\right)$, choose a closed halfspace $H_{i} \subset \mathbb{R}^{2}$ such that $\partial H_{i}$ is parallel to a ray in $\partial P_{i}$ (note that $P_{i}$ has only finitely many vertices), $\operatorname{spt}\left(\alpha_{i}\right) \subset \tilde{P}_{i}:=P_{i} \cap H_{i}$, and $\partial H_{i} \cap \operatorname{spt}\left(\alpha_{i}\right) \neq \emptyset$. Possibly $\tilde{P}_{i}=P_{i}$. Now there exists a ray $\rho_{i} \subset \partial \tilde{P}_{i}$ with vertex $y_{i}$ such that $\rho_{i} \cap \operatorname{spt}\left(\alpha_{i}\right)=\left\{y_{i}\right\}$. Let $\Sigma_{i} \subset \tilde{P}_{i}$ be the sector with vertex $y_{i}$ and $\rho_{i} \subset \partial \Sigma_{i}$ whose angle $\beta_{i}$ is maximal subject to the conditions $\beta_{i} \leq \alpha_{i}\left(y_{i}\right)$ and $\operatorname{int}\left(\Sigma_{i}\right) \cap \operatorname{spt}\left(\alpha_{i}\right)=\emptyset$. Finally, define

$$
P_{i+1}:=\operatorname{cl}\left(\tilde{P}_{i} \backslash \Sigma_{i}\right) \quad \text { and } \quad \alpha_{i+1}:=\alpha_{i}-\beta_{i} \delta_{y_{i}} .
$$

It is readily checked that $\operatorname{spt}\left(\alpha_{i+1}\right) \subset P_{i+1}$ and $0<\alpha_{i+1}\left(P_{i+1}\right) \leq \pi-\tau\left(\partial P_{i+1}\right)$ except in case $\alpha_{i+1}=0$ in which the process terminates, i.e., $i=k$. The sectors $\Sigma_{1}, \Sigma_{2}, \ldots$ constructed by this iterative procedure have pairwise disjoint interiors, and their vertices $y_{1}, y_{2}, \ldots$ belong to $\operatorname{spt}(\alpha)$. If $y_{i}=y_{j}$ for some $i \neq j$, then by construction there is a sector $\Sigma_{k}$ between $\Sigma_{i}$ and $\Sigma_{j}$, i.e., contained in the convex hull of $\Sigma_{i} \cup \Sigma_{j}$. Hence, there are at most $n:=\#(\operatorname{spt}(\alpha))$ sectors with the same vertex and at most $n^{2}$ sectors in total. This shows that the procedure indeed stops after finitely many iterations (a precise estimate is $k \leq 2 n-1$ ). The assertion $\sum_{y_{i}=x} \beta_{i}=\alpha(x)$ for every $x \in \operatorname{spt}(\alpha)$ follows since $\beta_{i} \leq \alpha_{i}\left(y_{i}\right)$, $\alpha_{i+1}=\alpha_{i}-\beta_{i} \delta_{y_{i}}$, and $\alpha_{k+1} \equiv 0$.

It remains to consider the case $P=\mathbb{R}^{2}$. In this case it suffices to find a line $l \subset \mathbb{R}^{2}$ with $\alpha\left(U^{\prime}\right), \alpha\left(U^{\prime \prime}\right) \leq \alpha\left(\mathbb{R}^{2}\right) / 2$ for the two open halfspaces $U^{\prime}, U^{\prime \prime}$ bounded by $l$. Then there exist measures $\alpha^{\prime}, \alpha^{\prime \prime} \geq 0$ on $\mathbb{R}^{2}$ with $\alpha^{\prime}+\alpha^{\prime \prime}=\alpha$, spt $\left(\alpha^{\prime}\right) \subset$ $P^{\prime}:=\operatorname{cl}\left(U^{\prime}\right), \operatorname{spt}\left(\alpha^{\prime \prime}\right) \subset P^{\prime \prime}:=\operatorname{cl}\left(U^{\prime \prime}\right)$, and $\alpha^{\prime}\left(P^{\prime}\right)=\alpha^{\prime \prime}\left(P^{\prime \prime}\right)=\alpha\left(\mathbb{R}^{2}\right) / 2 \leq \pi$. The result for $\left(\mathbb{R}^{2}, \alpha\right)$ now follows by applying the first part of the proof to both $\left(P^{\prime}, \alpha^{\prime}\right)$ and $\left(P^{\prime \prime}, \alpha^{\prime \prime}\right)$. A line $l$ as above exists through any given point in $\mathbb{R}^{2}$, or parallel to any given line, as is shown by simple continuity arguments.

Now we proceed to the choice of sectors on polyhedral halfplanes.

Proposition 5.2. Let $P$ be a polyhedral halfplane with boundary $\partial P$ and only finitely many interior and boundary vertices. Suppose that the total angle of every interior vertex $x$ of $P$ satisfies $\lambda(x)>\pi$. Denote by $v=\mu+\tau$ the total measure of $P$ and suppose $\alpha \geq 0$ is a measure on $P$ with finite support $\operatorname{spt}(\alpha)$ such that

$$
\alpha \geq v^{-} \text {and } 0<\alpha(P) \leq \pi-v(P) .
$$

Then there exist sectors $\Sigma_{i}=\left\{(r \cos (\theta), r \sin (\theta)): r \geq 0,0 \leq \theta \leq \beta_{i}\right\}$ in $\mathbb{R}^{2}$ and arcwise isometric embeddings $\sigma_{i}: \Sigma_{i} \rightarrow P$, for $i=1,2, \ldots$ up to some finite index $k$, such that $\sigma_{i}(0) \in \operatorname{spt}(\alpha), \sigma_{i}\left(\operatorname{int}\left(\Sigma_{i}\right)\right) \cap \sigma_{j}\left(\operatorname{int}\left(\Sigma_{j}\right)\right)=\emptyset$ for $i \neq j$, and $\sum_{\sigma_{i}(0)=x} \beta_{i}=\alpha(x)$ for every $x \in \operatorname{spt}(\alpha)$. 
Here we say that $\sigma_{i}: \Sigma_{i} \rightarrow P$ is an arcwise isometric embedding if $\sigma_{i}$ is a homeomorphism onto its image with the property that it preserves the length of all rectifiable curves, i.e. $L\left(\sigma_{i} \circ c\right)=L(c)$ for every such curve $c$ in $\Sigma_{i}$. This means that $\sigma_{i}$ is an isometric embedding with respect to the induced intrinsic metric on $\sigma_{i}\left(\Sigma_{i}\right) \subset P$. Note further that the assumptions of the proposition imply $\nu(P)<\pi$ and

$$
v^{+}(P)=v(P)+v^{-}(P) \leq v(P)+\alpha(P) \leq \pi .
$$

Proof. The plan of the proof is as follows. Starting with $P_{1}:=P, v_{1}:=v$, $\alpha_{1}:=\alpha$ we will construct a finite sequence

$$
P_{1} \supset \tilde{P}_{1} \supset P_{2} \supset \tilde{P}_{2} \supset \ldots
$$

of polyhedral halfplanes with total measures $v_{1}, \tilde{v}_{1}, v_{2}, \tilde{v}_{2}, \ldots$, respectively. For each $i=1,2, \ldots$ we will define a measure $0 \leq \alpha_{i} \leq \alpha$, with support $\operatorname{spt}\left(\alpha_{i}\right) \subset$ $\tilde{P}_{i} \subset P_{i}$, such that

$$
\begin{array}{ll}
\alpha_{i} \geq v_{i}^{-}, & 0<\alpha_{i}\left(P_{i}\right) \leq \pi-v_{i}\left(P_{i}\right), \\
\alpha_{i} \geq \tilde{v}_{i}^{-}, & 0<\alpha_{i}\left(\tilde{P}_{i}\right) \leq \pi-\tilde{v}_{i}\left(\tilde{P}_{i}\right) .
\end{array}
$$

$\tilde{P}_{i}$ will be obtained from $P_{i}$ by cutting off some flat strips along the boundary of $P_{i}$. The image of the embedding $\sigma_{i}: \Sigma_{i} \rightarrow P$ will be contained in $\tilde{P}_{i}$, and the procedure will terminate if $\alpha_{i+1}:=\alpha_{i}-\beta_{i} \delta_{\sigma_{i}(0)}=0$. In the opposite case, $P_{i+1}$ will be defined as the closure of $\tilde{P}_{i} \backslash \sigma_{i}\left(\Sigma_{i}\right)$. Throughout the proof $\partial$ and int refer to the boundary and interior of polyhedral surfaces (rather than to the topological boundary and interior). Moreover, in the proof we will denote by $[x, y]$ a not necessarily minimizing geodesic segment with endpoints $x$ and $y$.

Part I. Suppose we are given $P_{i}, v_{i}, \alpha_{i}$ satisfying (4). We construct $\tilde{P}_{i}, \tilde{v}_{i}$ with $\operatorname{spt}\left(\alpha_{i}\right) \subset \tilde{P}_{i} \subset P_{i}$,

$$
\tilde{v}_{i}^{-} \leq v_{i}^{-}, \quad \tilde{v}_{i}\left(\tilde{P}_{i}\right)=v_{i}\left(P_{i}\right),
$$

and the property that there exists a geodesic ray $\rho_{i} \subset \partial \tilde{P}_{i}$ with vertex $y_{i}$ and $\rho_{i} \cap \operatorname{spt}\left(\tilde{v}_{i}\right) \subset\left\{y_{i}\right\}=\rho_{i} \cap \operatorname{spt}\left(\alpha_{i}\right)$. Clearly (4) and (6) imply (5).

$\tilde{P}_{i}$ will be obtained via a finite sequence

$$
P_{i} \supset P_{i 0} \supset P_{i 1} \supset \cdots \supset P_{i n_{i}}=: \tilde{P}_{i}
$$

of polyhedral halfplanes for some index $n_{i} \geq 0$. The corresponding total measures will satisfy $v_{i}=v_{i 0}$ and

$$
v_{i 0}^{-} \geq v_{i 1}^{-} \geq \ldots \geq v_{i n_{i}}^{-}, \quad v_{i 0}\left(P_{i 0}\right)=v_{i 1}\left(P_{i 1}\right)=\ldots=v_{i n_{i}}\left(P_{i n_{i}}\right) .
$$

If $\partial P_{i} \cap\left(\operatorname{spt}\left(v_{i}\right) \cup \operatorname{spt}\left(\alpha_{i}\right)\right) \neq \emptyset$, then $P_{i 0}:=P_{i}$. If $\partial P_{i} \cap\left(\operatorname{spt}\left(v_{i}\right) \cup \operatorname{spt}\left(\alpha_{i}\right)\right)=\emptyset$, then there exists a closed set $F_{i 0} \subset P_{i}$ with $\partial P_{i} \subset F_{i 0}$ which, when equipped with 
its induced intrinsic metric, is isometric to a flat strip $\mathbb{R} \times\left[0, b_{i 0}\right]$; moreover, $F_{i 0}$ is chosen so that

$$
\emptyset \neq F_{i 0} \cap\left(\operatorname{spt}\left(v_{i}\right) \cup \operatorname{spt}\left(\alpha_{i}\right)\right) \subset \partial F_{i 0} .
$$

The set $P_{i 0}:=\operatorname{cl}\left(P_{i} \backslash F_{i 0}\right)$, equipped with the metric induced from $P_{i}$, is a polyhedral halfplane. In either case $v_{i 0}=v_{i}, \operatorname{spt}\left(\alpha_{i}\right) \subset P_{i 0}$, and $\partial P_{i 0} \cap\left(\operatorname{spt}\left(v_{i 0}\right) \cup\right.$ $\left.\operatorname{spt}\left(\alpha_{i}\right)\right) \neq \emptyset$.

Now suppose we are given, for some index $j \geq 0$, a polyhedral halfplane $P_{i j} \subset P_{i}$ with $\operatorname{spt}\left(\alpha_{i}\right) \subset P_{i j}$,

$$
v_{i j}^{-} \leq v_{i}^{-}, \quad v_{i j}\left(P_{i j}\right)=v_{i}\left(P_{i}\right),
$$

and $\partial P_{i j} \cap\left(\operatorname{spt}\left(v_{i j}\right) \cup \operatorname{spt}\left(\alpha_{i}\right)\right) \neq \emptyset$. If there exists a geodesic ray $\rho_{i} \subset \partial P_{i j}$ with vertex $y_{i}$ and $\rho_{i} \cap \operatorname{spt}\left(v_{i j}\right) \subset\left\{y_{i}\right\}=\rho_{i} \cap \operatorname{spt}\left(\alpha_{i}\right)$, then we put $\tilde{P}_{i}:=P_{i j}$, i.e., $j=n_{i}$, and we are done with Part I of the proof. If there is no such ray, we construct $P_{i, j+1}$ as follows. We have $\operatorname{spt}\left(v_{i j}\right) \cap \partial P_{i j} \neq \emptyset$. Moreover, since $\alpha_{i} \geq v_{i}^{-} \geq v_{i j}^{-}$, it follows that there exists a geodesic ray $\rho_{i j} \subset \partial P_{i j}$ with vertex $y_{i j}$ such that $\rho_{i j}$ is disjoint from $\operatorname{spt}\left(\alpha_{i}\right) \supset \operatorname{spt}\left(v_{i j}^{-}\right)$and meets $\operatorname{spt}\left(v_{i j}^{+}\right)$exactly in $y_{i j}$. By symmetry there is another such ray $\rho_{i j}^{\prime} \subset \partial P_{i j}$ with vertex $y_{i j}^{\prime} \in \operatorname{spt}\left(v_{i j}^{+}\right)$; this fact will be used below. The link of $P_{i j}$ at $y_{i j}$ satisfies $\lambda_{P_{i j}}\left(y_{i j}\right)=\pi-v_{i j}\left(y_{i j}\right)<\pi$. Then there exist a nontrivial geodesic segment $\left[y_{i j}, z_{i j}\right] \subset \partial P_{i j}$ with $\rho_{i j} \cap\left[y_{i j}, z_{i j}\right]=\left\{y_{i j}\right\}$ and a closed set $F_{i, j+1} \subset P_{i j}$ with $\rho_{i j} \cup\left[y_{i j}, z_{i j}\right] \subset F_{i, j+1}$ which, when equipped with its induced intrinsic metric, is isometric to the convex hull of two disjoint parallel rays in $\mathbb{R}^{2},\left[y_{i j}, z_{i j}\right]$ being isometric to the compact edge of this convex hull; moreover, $F_{i, j+1}$ is chosen so that

$$
\emptyset \neq F_{i, j+1} \cap\left(\left(\operatorname{spt}\left(v_{i j}\right) \backslash\left\{y_{i j}\right\}\right) \cup \operatorname{spt}\left(\alpha_{i}\right)\right) \subset \partial F_{i, j+1} .
$$

We put $P_{i, j+1}:=\operatorname{cl}\left(P_{i j} \backslash F_{i, j+1}\right)$ and equip this set with the metric induced from $P_{i j}$. We need to show that $P_{i, j+1}$ is a polyhedral halfplane. The relations (7) and (4) imply $v_{i j}\left(P_{i j}\right)<\pi$ and $v_{i j}^{+}\left(P_{i j}\right) \leq \pi$ (compare (3)). Hence, $P_{i, j+1} \neq \emptyset$ for if otherwise $v_{i j}\left(P_{i j}\right)=\pi$. Furthermore, the connected component of $F_{i, j+1} \cap \partial P_{i j}$ containing $\rho_{i j} \cup\left[y_{i j}, z_{i j}\right]$ coincides with $\rho_{i j} \cup\left[y_{i j}, z_{i j}\right]$ for if otherwise $v_{i j}^{+}\left(P_{i j}\right) \geq$ $v_{i j}^{+}\left(y_{i j}\right)+v_{i j}^{+}\left(z_{i j}\right)+v_{i j}^{+}\left(y_{i j}^{\prime}\right)=\pi+v_{i j}^{+}\left(y_{i j}^{\prime}\right)>\pi$ for the point $y_{i j}^{\prime} \in \operatorname{spt}\left(v_{i j}^{+}\right)$mentioned above. We claim that in fact $F_{i, j+1} \cap \partial P_{i j}=\rho_{i j} \cup\left[y_{i j}, z_{i j}\right]$. If not, then $P_{i, j+1}$ contains a polyhedral disk $\bar{D}$ with $\partial \bar{D} \subset F_{i, j+1} \cup \partial P_{i j}$ and $\bar{D} \cap F_{i, j+1}=$ $\left[z_{i j}, \bar{z}\right]$ for some $\bar{z} \neq z_{i j}$. The total measure $\bar{v}$ of $\bar{D}$ satisfies $\bar{v}\left(z_{i j}\right)=v_{i j}\left(z_{i j}\right)+$ $v_{i j}\left(y_{i j}\right)$ and $\bar{v}^{+}(\bar{D} \backslash\{\bar{z}\}) \leq v_{i j}^{+}\left(P_{i j}\right) \leq \pi$, while $\bar{v}(\bar{D})=2 \pi$ by the Gauss-Bonnet formula. Hence, $\bar{v}(\bar{z}) \geq \pi$. This contradiction proves the claim. It follows that $P_{i, j+1}$ is a polyhedral halfplane. We have $v_{i, j+1}=v_{i j}+v_{i j}\left(y_{i j}\right)\left(\delta_{z_{i j}}-\delta_{y_{i j}}\right)$, therefore

$$
v_{i, j+1}^{-} \leq v_{i j}^{-} \leq v_{i}^{-} \quad \text { and } \quad v_{i, j+1}\left(P_{i, j+1}\right)=v_{i j}\left(P_{i j}\right)=v_{i}\left(P_{i}\right)
$$


due to (7). By construction, $\operatorname{spt}\left(\alpha_{i}\right) \subset P_{i, j+1}$. Since $\alpha_{i}\left(z_{i j}\right) \geq v_{i j}^{-}\left(z_{i j}\right)$, we see that $\partial P_{i, j+1} \cap\left(\operatorname{spt}\left(v_{i, j+1}\right) \cup \operatorname{spt}\left(\alpha_{i}\right)\right) \neq \varnothing$ as for $P_{i j}$.

We need to show that this iterative process terminates, i.e. produces the desired polyhedral halfplane $P_{i n_{i}}=\tilde{P}_{i}$. Suppose $P_{i, j+1}$ is not yet the desired surface. If $z_{i j} \in \operatorname{spt}\left(v_{i j}\right)$, then $\#\left(\operatorname{spt}\left(v_{i, j+1}\right)\right)<\#\left(\operatorname{spt}\left(v_{i j}\right)\right)$ (the total number of vertices) and $\#\left(\operatorname{spt}\left(v_{i, j+1}\right) \cap \operatorname{int}\left(P_{i, j+1}\right)\right) \leq \#\left(\operatorname{spt}\left(v_{i j}\right) \cap \operatorname{int}\left(P_{i j}\right)\right)$ (the number of interior vertices). If $z_{i j} \notin \operatorname{spt}\left(v_{i j}\right)$, then $\#\left(\operatorname{spt}\left(v_{i, j+1}\right)\right)=\#\left(\operatorname{spt}\left(v_{i j}\right)\right)$ and $\#\left(\operatorname{spt}\left(v_{i, j+1}\right) \cap \operatorname{int}\left(P_{i, j+1}\right)\right)<\#\left(\operatorname{spt}\left(v_{i j}\right) \cap \operatorname{int}\left(P_{i j}\right)\right)$. Since $v_{i 0}=v_{i}$ it follows that $n_{i} \leq 2 \#\left(\operatorname{spt}\left(v_{i}\right)\right)$.

Part II. We are given $\tilde{P}_{i}, \tilde{v}_{i}, \alpha_{i}$ satisfying (5), and there exists a geodesic ray $\rho_{i} \subset \partial \tilde{P}_{i}$ with vertex $y_{i}$ such that $\rho_{i} \cap \operatorname{spt}\left(\tilde{v}_{i}\right) \subset\left\{y_{i}\right\}=\rho_{i} \cap \operatorname{spt}\left(\alpha_{i}\right)$. We construct $\sigma_{i}: \Sigma_{i} \rightarrow \tilde{P}_{i}$ and $P_{i+1}, \alpha_{i+1}$ as follows.

Let $\Sigma_{i}:=\left\{(r \cos (\theta), r \sin (\theta)): r \geq 0,0 \leq \theta \leq \beta_{i}\right\}$ be the sector in $\mathbb{R}^{2}$ whose angle $\beta_{i} \in(0, \pi]$ is maximal subject to the following conditions: There exists an arcwise isometric embedding $\sigma_{i}: \Sigma_{i} \rightarrow \tilde{P}_{i}$ with $\sigma_{i}(0)=y_{i}, \sigma_{i}(\{(r, 0)$ : $r \geq 0\})=\rho_{i}, \sigma_{i}\left(\operatorname{int}\left(\Sigma_{i}\right)\right) \cap \operatorname{spt}\left(\alpha_{i}\right)=\emptyset$, and $\beta_{i} \leq \alpha_{i}\left(y_{i}\right)$. (Such a maximal embedded sector exists due to the properties of $\rho_{i}$ and the fact that the link of every interior vertex of $\tilde{P}_{i} \subset P$ is $>\pi$.) We put

$$
P_{i+1}:=\operatorname{cl}\left(\tilde{P}_{i} \backslash \sigma_{i}\left(\Sigma_{i}\right)\right), \quad \alpha_{i+1}:=\alpha_{i}-\beta_{i} \delta_{y_{i}}
$$

and equip $P_{i+1}$ with the metric induced from $\tilde{P}_{i}$. In case $\alpha_{i+1}=0$ all sectors are selected and the process terminates, i.e., $i=k$. In case $\alpha_{i+1} \neq 0$ we need to show that $P_{i+1}$ is a polyhedral halfplane, $\operatorname{spt}\left(\alpha_{i+1}\right) \subset P_{i+1}$,

$$
\alpha_{i+1} \geq v_{i+1}^{-}, \quad \text { and } \quad \alpha_{i+1}\left(P_{i+1}\right) \leq \pi-v_{i+1}\left(P_{i+1}\right) .
$$

We consider the following two cases.

Case I. $\sigma_{i}\left(\Sigma_{i}\right) \cap \partial \tilde{P}_{i}=\rho_{i}$. Then $P_{i+1}$ is a polyhedral halfplane, $\operatorname{spt}\left(\alpha_{i+1}\right) \subset P_{i+1}$, and $v_{i+1}=\tilde{v}_{i}+\beta_{i} \delta_{y_{i}}$, as is easily checked. Together with (5) this gives

$$
\alpha_{i+1}\left(P_{i+1}\right)=\alpha_{i}\left(\tilde{P}_{i}\right)-\beta_{i} \leq \pi-\tilde{v}_{i}\left(\tilde{P}_{i}\right)-\beta_{i}=\pi-v_{i+1}\left(P_{i+1}\right) .
$$

If $v_{i+1}^{-}\left(y_{i}\right)>0$, then $\alpha_{i+1}\left(y_{i}\right)=\alpha_{i}\left(y_{i}\right)-\beta_{i} \geq \tilde{v}_{i}^{-}\left(y_{i}\right)-\beta_{i}=v_{i+1}^{-}\left(y_{i}\right)$. Hence, (8) holds.

Case II. $\sigma_{i}\left(\Sigma_{i}\right) \cap \partial \tilde{P}_{i}=\rho_{i} \cup\left[y_{i}, z_{i}\right]$ for some nontrivial segment $\left[y_{i}, z_{i}\right]$ with $\left[y_{i}, z_{i}\right] \cap \rho_{i}=\left\{y_{i}\right\}$. Again, $P_{i+1}$ is a polyhedral halfplane. However, we now have $y_{i} \notin P_{i+1}$ and it is not immediately clear that $\operatorname{spt}\left(\alpha_{i+1}\right) \subset P_{i+1}$. Using (5) we observe that 


$$
\begin{aligned}
\alpha_{i}\left(\tilde{P}_{i} \backslash \operatorname{spt}\left(\tilde{v}_{i}^{-}\right)\right) & =\alpha_{i}\left(\tilde{P}_{i}\right)-\alpha_{i}\left(\operatorname{spt}\left(\tilde{v}_{i}^{-}\right)\right) \\
& \leq \alpha_{i}\left(\tilde{P}_{i}\right)-\tilde{v}_{i}^{-}\left(\tilde{P}_{i}\right) \\
& \leq \pi-\tilde{v}_{i}^{+}\left(\tilde{P}_{i}\right) \\
& \leq \pi-\tilde{v}_{i}^{+}\left(y_{i}\right) \\
& =\lambda_{\tilde{P}_{i}}\left(y_{i}\right)
\end{aligned}
$$

the link of $\tilde{P}_{i}$ at $y_{i}$. Since $\lambda_{\tilde{P}_{i}}\left(y_{i}\right)=\beta_{i} \leq \alpha_{i}\left(y_{i}\right)$ and $\operatorname{spt}\left(\tilde{v}_{i}^{-}\right) \cap\left[y_{i}, z_{i}\right] \backslash\left\{z_{i}\right\}=\emptyset$ it follows that $\alpha_{i} \mid\left(\left[y_{i}, z_{i}\right] \backslash\left\{z_{i}\right\}\right)=\beta_{i} \delta_{y_{i}}$, hence, $\operatorname{spt}\left(\alpha_{i+1}\right) \subset P_{i+1}$. The total measure satisfies $v_{i+1}=\tilde{v}_{i}-\tilde{v}_{i}\left(y_{i}\right) \delta_{y_{i}}+\pi \delta_{z_{i}}$, where $\tilde{v}_{i}\left(y_{i}\right)=\pi-\lambda_{\tilde{P}_{i}}\left(y_{i}\right)=\pi-\beta_{i}$. We get again (9). By (5), $v_{i+1}^{-}\left(z_{i}\right) \leq \tilde{v}_{i}^{-}\left(z_{i}\right) \leq \alpha_{i}\left(z_{i}\right)=\alpha_{i+1}\left(z_{i}\right)$. Thus, (8) holds.

We show that these two cases cover all possibilities. If $\sigma_{i}\left(\Sigma_{i}\right) \cap \partial \tilde{P}_{i}=\partial \tilde{P}_{i}$, i.e. $\sigma_{i}\left(\Sigma_{i}\right)=\tilde{P}_{i}$, then by the same argument as in Case II we would get $\alpha_{i}=\beta_{i} \delta_{y_{i}}$, hence $\alpha_{i+1} \equiv 0$, in contradiction to $\alpha_{i+1} \not \equiv 0$. It remains to consider the case that $\sigma_{i}\left(\Sigma_{i}\right) \cap \partial \tilde{P}_{i}$ is disconnected. Then $P_{i+1}$ contains a polyhedral disk $\tilde{D}$ with $\partial \tilde{D} \subset \sigma_{i}\left(\Sigma_{i}\right) \cup \partial \tilde{P}_{i}$ such that $\tilde{D} \cap \sigma_{i}\left(\Sigma_{i}\right)=\left[z_{i}, \tilde{z}\right]$ is a nontrivial segment and $z_{i}$ belongs to the component of $\sigma_{i}\left(\Sigma_{i}\right) \cap \partial \tilde{P}_{i}$ containing $\rho_{i}$. (Possibly $z_{i}=y_{i}$.) Denote by $\tilde{v}$ the total measure of $\tilde{D}$. Note that in Cases I and II above, we have $v_{i+1}^{+}\left(P_{i+1}\right) \leq \pi$ due to (8). The same arguments show that $\tilde{v}^{+}(\tilde{D} \backslash\{\tilde{z}\}) \leq \pi$, while $\tilde{v}(\tilde{D})=2 \pi$ by the Gauss-Bonnet formula. Hence, $\tilde{v}(\tilde{z}) \geq \pi$, which is impossible. This concludes Part II of the proof.

It remains to show that the whole procedure terminates after finitely many iterations. Let $N_{i}:=\#\left(\left(\operatorname{spt}\left(\alpha_{i}\right) \cup \operatorname{spt}\left(v_{i}\right)\right) \cap \operatorname{int}\left(P_{i}\right)\right)$. For $i=1,2, \ldots$ we have $\alpha_{i+1} \leq \alpha_{i}$ and $N_{i+1} \leq N_{i}$. In addition, at least one of the following assertions holds: $\alpha_{i+1}\left(P_{i+1}\right)=\alpha_{i}\left(P_{i}\right)-\pi$ (if $\left.\beta_{i}=\pi\right)$, or \# $\left(\operatorname{spt}\left(\alpha_{i+1}\right)\right)<\#\left(\operatorname{spt}\left(\alpha_{i}\right)\right)$ (if $\beta_{i}=\alpha_{i}\left(y_{i}\right)$, in particular in Case II), or $N_{i+1}<N_{i}$ (if $\beta_{i}<\alpha_{i}\left(y_{i}\right)$ and $\beta_{i}<\pi$ ). It follows that $\alpha_{k+1} \equiv 0$ for some finite index $k$.

\section{Splitting the surface}

Our next goal is to prove an analog of Proposition 5.2 for polyhedral planes rather than halfplanes. As in Lemma 5.1, the idea is to split the surface into two parts and to apply the previous result to each of them. To obtain a suitable splitting of polyhedral planes, we first prove a corresponding result in the smooth case which may be of independent interest. In fact, the following statement is a partial strengthening of Theorem 2 in [Ba2].

Proposition 6.1. Let $X$ be a smooth complete Riemannian surface homeomorphic to the plane with Gaussian curvature K. Suppose that $\int_{X} K^{+}<2 \pi$. Let $h$ be a continuous non-negative function on $X$ with $\int_{X} h<\infty$. Then there exists a properly embedded, complete geodesic line $l \subset X$ such that 


$$
\begin{aligned}
\int_{U^{\prime}} K^{+}=\int_{U^{\prime \prime}} K^{+} & =\int_{X} K^{+} / 2 \\
\text { and } \quad \int_{U^{\prime}} h=\int_{U^{\prime \prime}} h & =\int_{X} h / 2,
\end{aligned}
$$

where $U^{\prime}, U^{\prime \prime}$ denote the connected components of $X \backslash$ l. Moreover, if $c: \mathbb{R} \rightarrow X$ is a unit speed parameterization of $l$, then

$$
d(c(r), c(s)) \geq \cos \left(\int_{X} K^{+} / 4\right)|r-s|
$$

for all $r, s \in \mathbb{R}$. In particular, $c$ is a bi-Lipschitz embedding.

The constant $\cos \left(\int_{X} K^{+} / 4\right)>0$ is optimal for rotationally symmetric surfaces of non-negative curvature.

Proof. For simplicity we prove this only in case $\operatorname{spt}\left(K^{+}\right) \cup \operatorname{spt}(h)$ is compact; this is all we need for the proof of Proposition 6.2. A straightforward modification of our argument yields the general result.

We assume also that $K^{+}, h \not \equiv 0$. (In case $K \leq 0$ one easily finds a minimizing geodesic line $l$ with $\int_{U^{\prime}} h=\int_{U^{\prime \prime}} h=\int_{X} h / 2$, in case $K^{+} \not \equiv 0$ and $h \equiv 0$ one can apply the following proof for $h:=K^{+}$.) We first observe that there exists a compact topological disk $\tilde{D} \subset X$ such that its boundary $\partial \tilde{D}$ is $C^{1}$, $\operatorname{spt}\left(K^{+}\right) \cup \operatorname{spt}(h) \subset \tilde{D}$, and $\tilde{D}$ is totally convex: every geodesic segment in $X$ (minimizing or not) with endpoints in $\tilde{D}$ lies in $\tilde{D}$. For instance, the totally convex hull of a closed metric ball containing $\operatorname{spt}\left(K^{+}\right) \cup \operatorname{spt}(h)$ has this property (cf. [Ba1, Section 2]). We note further that every non-constant geodesic $c: \mathbb{R} \rightarrow X$ is proper due to the assumption $\int_{X} K^{+}<2 \pi$ and the Gauss-Bonnet formula. Moreover, in case $c$ is not injective, there exist uniquely determined numbers $a<b$ such that $c \mid[a, b)$ is injective and $c(a)=c(b)$, i.e., $c([a, b])$ is a simple geodesic loop. The two rays $c((-\infty, a))$ and $c((b, \infty))$ are embedded and disjoint from $c([a, b])$, but they may intersect each other (see [Re, p. 149] or [Ba1, Corollary 2]).

We define two continuous functions $\tilde{k}^{+}, \tilde{k}^{-}: S \tilde{D} \rightarrow[0,1]$ on the unit sphere bundle $S \tilde{D}$ of $\tilde{D}$ as follows. Given a unit vector $v \in S_{y} \tilde{D}$ at $y$, consider the complete geodesic line $l(v) \subset X$ through $y$ perpendicular to $v$. We select some connected components of $X \backslash l(v)$ such that their union $U(v)$ satisfies $\partial U(v)=l(v)$; there are exactly two possible (complementary) selections. In case $l(v)$ has no self-intersections we choose $U(v)$ so that $v$ points to $U(v)$. In the opposite case we fix a unit speed parameterization $c_{v}$ of $l(v)$ and a continuous unit normal vector field $n_{v}$ along $c_{v}$ with $n_{v}(0)=v$. Then we choose $U(v)$ so that $n_{v}\left(\left(a_{v}+b_{v}\right) / 2\right)$ points to $U(v)$ for the unique simple geodesic loop $c_{v}\left(\left[a_{v}, b_{v}\right]\right)$ of $l(v)$. We define $\tilde{k}^{+}, \tilde{k}^{-}: S \tilde{D} \rightarrow[0,1]$ by

$$
\tilde{k}^{+}(v):=\frac{\int_{U(v)} K^{+}}{\int_{X} K^{+}}, \quad \tilde{k}^{-}(v):=\frac{\int_{U(v)} h}{\int_{X} h} .
$$

Clearly $\tilde{k}^{ \pm}(v)+\tilde{k}^{ \pm}(-v)=1$ for all $v \in S \tilde{D}$. From the total convexity of $\tilde{D}$ it follows that $\tilde{k}^{ \pm}(v)=1$ if $y \in \partial \tilde{D}$ and $v \in S_{y} \tilde{D}$ is the uniquely determined 
inward normal. (If $l(v)$ has self-intersections, then the unique simple geodesic loop of $l(v)$ must enclose $\tilde{D}$ since $\operatorname{spt}\left(K^{+}\right) \subset \tilde{D}$.) To obtain the desired splitting of $\int_{X} K^{+}$and $\int_{X} h$ it suffices to find a vector $v_{0} \in S \tilde{D}$ such that

$$
\tilde{k}^{+}\left(v_{0}\right)=\tilde{k}^{-}\left(v_{0}\right)=1 / 2 .
$$

We claim that whenever $v_{0}$ is a vector with this property, then the corresponding geodesic line $l\left(v_{0}\right)$ is embedded. Suppose it is not. Denote by $B \subset X$ the open disk bounded by the unique simple geodesic loop in $l\left(v_{0}\right)$ and by $\beta>0$ the interior angle at the vertex of the loop. By the Gauss-Bonnet formula, $\int_{B} K=\pi+\beta$. On the other hand, we have either $B \subset U\left(v_{0}\right)$ or $B \subset X \backslash U\left(v_{0}\right)$ and therefore $\int_{B} K \leq \int_{B} K^{+} \leq \int_{X} K^{+} / 2<\pi$ since $\tilde{k}^{+}\left(v_{0}\right)=1 / 2$; a contradiction.

To find $v_{0}$ we first choose a diffeomorphism $\varphi: D \rightarrow \tilde{D}$ from the closed unit disk $D \subset \mathbb{R}^{2}$ onto $\tilde{D}$ with the property that $d \varphi_{u}(-u)$ is normal to $\partial \tilde{D}$ at $\varphi(u)$. We define two continuous functions $k^{+}, k^{-}$on the unit sphere bundle $S D=D \times \mathbb{S}^{1}$ of $D$ by

$$
k^{ \pm}(x, u)=\tilde{k}^{ \pm}\left(d \varphi_{x}(u) /\left|d \varphi_{x}(u)\right|\right) ;
$$

then $k^{ \pm}(u,-u)=1$ and $k^{ \pm}(x, u)+k^{ \pm}(x,-u)=1$ for all $u \in \mathbb{S}^{1}$ and $x \in D$. Finally, we define a homotopy $k: \mathbb{S}^{1} \times[0,1] \rightarrow \mathbb{R}^{2}$ by

$$
k(u, t):=\left(k^{+}(t u,-u)-1 / 2, k^{-}(t u,-u)-1 / 2\right) .
$$

We have $k(u, 0)=-k(-u, 0)$ and $k(u, 1)=(1 / 2,1 / 2)$ for all $u \in \mathbb{S}^{1}$. Then there exists a point $\left(u_{0}, t_{0}\right) \in \mathbb{S}^{1} \times[0,1]$ with $k\left(u_{0}, t_{0}\right)=(0,0)$, for if not, normalizing $k$ we would obtain a continuous map from $\mathbb{S}^{1}$ to itself that carries antipodal points to antipodal points and has zero mod 2 degree, in contradiction to the BorsukUlam theorem for $\mathbb{S}^{1}$ (cf. [GP, p. 93]). Now $v_{0}:=d \varphi_{t_{0} u_{0}}\left(-u_{0}\right) /\left|d \varphi_{t_{0} u_{0}}\left(-u_{0}\right)\right|$ is the desired unit vector.

Let $c: \mathbb{R} \rightarrow X$ be a unit speed parameterization of $l$. Denote by $d^{\prime}, d^{\prime \prime}$ the induced intrinsic metrics on $\operatorname{cl}\left(U^{\prime}\right)$ and $\operatorname{cl}\left(U^{\prime \prime}\right)$, respectively. To prove the last assertion of the proposition it suffices to show that $d^{\prime}(c(r), c(s)) \geq \cos \left(\int_{X} K^{+} / 4\right)|s-r|$ for all $r, s \in \mathbb{R}$. By symmetry the same estimate then holds for $d^{\prime \prime}$, and a simple subdivision argument yields the general case. Put

$$
w(r, s):=d^{\prime}(c(r), c(s)) /(s-r)
$$

for $r<s$. Note that $w \leq 1$ and $w(r, s)=1$ if $c \mid[r, s]$ is minimizing in $X$. Let $R>0$. There exists a minimum $\left(r_{0}, s_{0}\right)$ of $w \mid\left\{(r, s) \in \mathbb{R}^{2}:-R \leq r<s \leq R\right\}$. Pick a shortest unit speed curve $\gamma_{0}:\left[0, b_{0}\right] \rightarrow \operatorname{cl}\left(U^{\prime}\right)$ from $c\left(r_{0}\right)$ to $c\left(s_{0}\right)$, i.e., $b_{0}=$ $d^{\prime}\left(c\left(r_{0}\right), c\left(s_{0}\right)\right), \gamma_{0}(0)=c\left(r_{0}\right)$, and $\gamma_{0}\left(b_{0}\right)=c\left(s_{0}\right)$. Since $c$ is a geodesic it follows that $\gamma_{0}\left(\left[0, b_{0}\right]\right)$ is a geodesic arc which either coincides with $c\left(\left[r_{0}, s_{0}\right]\right)$ or intersects $l$ exactly in $\left\{c\left(r_{0}\right), c\left(s_{0}\right)\right\}$. In the first case we have $w\left(r_{0}, s_{0}\right)=1$ and hence $w(r, s)=1$ for $-R \leq r<s \leq R$. In the second case, denote by $B_{0} \subset U^{\prime}$ 
the open disk bounded by $c\left(\left[r_{0}, s_{0}\right]\right) \cup \gamma_{0}\left(\left[0, b_{0}\right]\right)$ and by $\alpha, \beta \in(0, \pi)$ the two interior angles at $c\left(r_{0}\right)$ and $c\left(s_{0}\right)$, respectively. The Gauss-Bonnet formula yields

$$
\alpha+\beta=\int_{B_{0}} K \leq \int_{B_{0}} K^{+} \leq \int_{U^{\prime}} K^{+}=\int_{X} K^{+} / 2 .
$$

By symmetry we may assume that $\alpha \leq \beta$, hence $\alpha \leq \int_{X} K^{+} / 4$. Now consider a smooth variation $\left\{\gamma_{t}\right\}_{-\epsilon<t<\epsilon}$ of $\gamma_{0}$ in $\operatorname{cl}\left(U^{\prime}\right)$ with endpoints $\gamma_{t}(0)=c\left(r_{0}+\right.$ $t), \gamma_{t}\left(b_{0}\right)=c\left(s_{0}\right)$. Since $L\left(\gamma_{t}\right) /\left(s_{0}-r_{0}-t\right) \geq w\left(r_{0}+t, s_{0}\right) \geq w\left(r_{0}, s_{0}\right)=$ $L\left(\gamma_{0}\right) /\left(s_{0}-r_{0}\right)$ for $t \geq 0$, we get

$$
0 \leq\left.\frac{d}{d t}\right|_{t=0}\left(\frac{L\left(\gamma_{t}\right)}{s_{0}-r_{0}-t}\right)=\frac{-\cos (\alpha)+w\left(r_{0}, s_{0}\right)}{s_{0}-r_{0}}
$$

by the first variation formula. Hence,

$$
w(r, s) \geq w\left(r_{0}, s_{0}\right) \geq \cos (\alpha) \geq \cos \left(\int_{X} K^{+} / 4\right)
$$

whenever $-R \leq r<s \leq R$. As this holds for all $R>0$, we conclude that $w \geq \cos \left(\int_{X} K^{+} / 4\right)$.

Now we derive an analog of Proposition 6.1 for polyhedral planes $X$. Any properly embedded, complete polygonal line $l \subset X$ gives rise to a splitting of $X$ into two polyhedral halfplanes $P^{\prime}, P^{\prime \prime}$, the closures of the connected components of $X \backslash l$, endowed with their induced intrinsic metrics. As mentioned earlier, the corresponding total measures satisfy $v_{P^{\prime}}+v_{P^{\prime \prime}}=\mu$ where $\mu$ denotes the integral curvature of $X$. If, in addition, $v_{P^{\prime}}^{+}+v_{P^{\prime \prime}}^{+}=\mu^{+}$, hence also $v_{P^{\prime}}^{-}+v_{P^{\prime \prime}}^{-}=\mu^{-}$, then $l$ will be called a properly embedded, complete quasigeodesic line. This additional assumption is equivalent to saying that $l$ is everywhere geodesic, i.e. locally distance minimizing, except at points where it hits $\operatorname{spt}\left(\mu^{+}\right)$; at such points it is required that the turns of $l=\partial P^{\prime}=\partial P^{\prime \prime}$ respective to both $P^{\prime}, P^{\prime \prime}$ are nonnegative.

Proposition 6.2. Let $X$ be a polyhedral plane with only finitely many vertices and $\mu^{+}(X)<2 \pi$. Let $\eta \geq 0$ be a measure on $X$ with finite support. Then there exist a properly embedded, complete quasigeodesic line $l \subset X$ and measures $\eta^{\prime}, \eta^{\prime \prime} \geq 0$ on $X$ such that $\eta^{\prime}+\eta^{\prime \prime}=\eta, \operatorname{spt}\left(\eta^{\prime}\right) \subset P^{\prime}, \operatorname{spt}\left(\eta^{\prime \prime}\right) \subset P^{\prime \prime}$,

$$
\begin{aligned}
v_{P^{\prime}}^{+}\left(P^{\prime}\right)=v_{P^{\prime \prime}}^{+}\left(P^{\prime \prime}\right) & =\mu^{+}(X) / 2 \\
\text { and } \quad \eta^{\prime}\left(P^{\prime}\right)=\eta^{\prime \prime}\left(P^{\prime \prime}\right) & =\eta(X) / 2,
\end{aligned}
$$

where $P^{\prime}, P^{\prime \prime}$ denote the closures of the connected components of $X \backslash l$, endowed with their induced intrinsic metrics, and $v_{P^{\prime}}, v_{P^{\prime \prime}}$ denote the corresponding total measures. Moreover, $\eta^{\prime}(x)=\eta(x) v_{P^{\prime}}(x) / \mu(x)$ and $\eta^{\prime \prime}(x)=\eta(x) v_{P^{\prime \prime}}(x) / \mu(x)$ for each vertex $x \in \operatorname{spt}(\mu)$. If $c: \mathbb{R} \rightarrow X$ is a unit speed parameterization of $l$, then

$$
d(c(r), c(s)) \geq \cos \left(\mu^{+}(X) / 4\right)|r-s|
$$

for all $r, s \in \mathbb{R}$. In particular, $c$ is a bi-Lipschitz embedding. 
For instance, if $\eta=\mu^{-}$, then $\eta^{\prime}=v_{P^{\prime}}^{-}$and $\eta^{\prime \prime}=v_{P^{\prime \prime}}^{-}$(note that $\eta(x)=0$ implies $\eta^{\prime}(x)=\eta^{\prime \prime}(x)=0$, and $\mu^{-}(x)=0$ implies $\left.v_{P^{\prime}}^{-}(x)=v_{P^{\prime \prime}}^{-}(x)=0\right)$.

Proof. The result follows from its smooth analog Proposition 6.1 by means of approximation. We assume that $\operatorname{spt}(\mu) \neq \varnothing$ and $\operatorname{spt}\left(\mu^{+}\right) \cup \operatorname{spt}(\eta) \neq \varnothing$. Let $\epsilon_{1}>\epsilon_{2}>\cdots>0$ be a sequence converging to 0 , such that $d\left(x, x^{\prime}\right)>2 \epsilon_{1}$ for every pair of distinct points $x, x^{\prime} \in \operatorname{spt}(\mu) \cup \operatorname{spt}(\eta)$. On $X \backslash \operatorname{spt}(\mu)$ we have a flat Riemannian metric $g$ whose induced distance function is the given metric $d$. We choose a sequence of smooth Riemannian metrics $g_{1}, g_{2}, \ldots$ on $X$ such that

(i) $g_{i}$ coincides with $g$ on $X \backslash \bigcup_{x \in \operatorname{spt}(\mu)} B\left(x, \epsilon_{i}\right)$,

(ii) for each $x \in \operatorname{spt}(\mu)$, the Gaussian curvature of $g_{i}$ satisfies $K_{i} \mid B\left(x, \epsilon_{i}\right) \geq 0$ if $\mu(x)>0$ and $K_{i} \mid B\left(x, \epsilon_{i}\right) \leq 0$ if $\mu(x)<0$,

(iii) for each $x \in \operatorname{spt}(\mu)$, the diameter of $B\left(x, \epsilon_{i}\right)$ in $\left(X, g_{i}\right)$ is $\leq 2 \epsilon_{i}$.

Here $B\left(x, \epsilon_{i}\right)$ denotes the open ball with respect to $d$. By the Gauss-Bonnet formula,

$$
\int_{B\left(x, \epsilon_{i}\right)} K_{i} d A_{i}=2 \pi-\int_{\partial B\left(x, \epsilon_{i}\right)} \kappa d s=2 \pi-\lambda(x)=\mu(x)
$$

for all $x \in \operatorname{spt}(\mu)$, where $d A_{i}$ is the area element with respect to $g_{i}$, and the geodesic curvature $\kappa$ and line element $d s$ of $\partial B\left(x, \epsilon_{i}\right)$ are the same for $g$ and for all $g_{i}$. The distance functions $d_{i}$ on $X$ induced by the $g_{i}$ converge uniformly to the given metric $d$ as $i \rightarrow \infty$. We choose continuous non-negative functions $h_{i}$ on $X$ such that $h_{i}$ vanishes on $X \backslash \bigcup_{x \in \operatorname{spt}(\eta)} B\left(x, \epsilon_{i}\right)$ and

$$
\int_{B\left(x, \epsilon_{i}\right)} h_{i} d A_{i}=\eta(x)
$$

for each $x \in \operatorname{spt}(\eta)$. Specifically, for each $x \in \operatorname{spt}(\mu) \cap \operatorname{spt}(\eta)$, we put $h_{i}:=$ $(\eta(x) / \mu(x)) K_{i}=(\eta(x) /|\mu(x)|) /\left|K_{i}\right|$ on $B\left(x, \epsilon_{i}\right)$.

Applying Proposition 6.1 to $\left(X, g_{i}, h_{i}\right)$ we obtain properly embedded geodesic lines $l_{i} \subset\left(X, g_{i}\right)$ such that

$$
\begin{aligned}
& \int_{U_{i}^{\prime}} K_{i}^{+} d A_{i}=\int_{U_{i}^{\prime \prime}} K_{i}^{+} d A_{i}=\int_{X} K_{i}^{+} d A_{i} / 2=\mu^{+}(X) / 2 \\
& \text { and } \int_{U_{i}^{\prime}} h_{i} d A_{i}=\int_{U_{i}^{\prime \prime}} h_{i} d A_{i}=\int_{X} h_{i} d A_{i} / 2=\eta(X) / 2 \text {, }
\end{aligned}
$$

where $U_{i}^{\prime}, U_{i}^{\prime \prime}$ denote the connected components of $X \backslash l_{i}$. For each $i$ we choose a unit speed parameterization $c_{i}: \mathbb{R} \rightarrow\left(X, g_{i}\right)$ of $l_{i}$ such that $c_{i}(0)$ belongs to some fixed compact set. Then

$$
\begin{aligned}
d_{i}\left(c_{i}(r), c_{i}(s)\right) & \geq \cos \left(\int_{X} K_{i}^{+} d A_{i} / 4\right)|r-s| \\
& =\cos \left(\mu^{+}(X) / 4\right)|r-s|
\end{aligned}
$$

for all $i$ and for all $r, s \in \mathbb{R}$. After passing to some subsequence, without changing notation, we may assume that the $c_{i}$ converge uniformly on compact sets to some curve $c: \mathbb{R} \rightarrow(X, d)$ satisfying

$$
\cos \left(\mu^{+}(X) / 4\right)|r-s| \leq d(c(r), c(s)) \leq|r-s|
$$


for all $r, s \in \mathbb{R}$. Denote by $l$ the trace of $c$ and by $U^{\prime}, U^{\prime \prime}$ the two connected components of $X \backslash l$. We may assume that on $U^{\prime} \cup U^{\prime \prime}=X \backslash l$, the characteristic functions of $U_{i}^{\prime}$ and $U_{i}^{\prime \prime}$ converge locally uniformly to those of $U^{\prime}$ and $U^{\prime \prime}$, respectively. Finally, due to (10) and (11) we may assume that the four limits

$$
\begin{array}{cc}
\lim _{i \rightarrow \infty} \int_{U_{i}^{\prime} \cap B\left(x, \epsilon_{i}\right)} K_{i} d A_{i}, & \lim _{i \rightarrow \infty} \int_{U_{i}^{\prime \prime} \cap B\left(x, \epsilon_{i}\right)} K_{i} d A_{i}, \\
\lim _{i \rightarrow \infty} \int_{U_{i}^{\prime} \cap B\left(x, \epsilon_{i}\right)} h_{i} d A_{i}, & \lim _{i \rightarrow \infty} \int_{U_{i}^{\prime \prime} \cap B\left(x, \epsilon_{i}\right)} h_{i} d A_{i}
\end{array}
$$

exist for all $x \in X$.

Since the $c_{i}$ are unit speed geodesics it follows that $c$ is a unit speed geodesic outside $\operatorname{spt}(\mu)$, hence, $c$ is a polygonal unit speed curve with vertices in $\operatorname{spt}(\mu)$. Denote by $P^{\prime}, P^{\prime \prime}$ the closures of $U^{\prime}, U^{\prime \prime}$, endowed with their induced intrinsic metrics, and by $v_{P^{\prime}}, v_{P^{\prime \prime}}$ the corresponding total measures with $v_{P^{\prime}}=0$ on $U^{\prime \prime}$, $v_{P^{\prime \prime}}=0$ on $U^{\prime}$. We have $\operatorname{spt}\left(v_{P^{\prime}}\right), \operatorname{spt}\left(v_{P^{\prime \prime}}\right) \subset \operatorname{spt}(\mu)$. We claim that

$$
\begin{gathered}
v_{P^{\prime}}(x)=\lim _{i \rightarrow \infty} \int_{U_{i}^{\prime} \cap B\left(x, \epsilon_{i}\right)} K_{i} d A_{i}, \\
v_{P^{\prime \prime}}(x)=\lim _{i \rightarrow \infty} \int_{U_{i}^{\prime \prime} \cap B\left(x, \epsilon_{i}\right)} K_{i} d A_{i}
\end{gathered}
$$

for all $x \in X$. We prove (14). If $x \in X \backslash\left(\operatorname{spt}(\mu) \cap P^{\prime}\right)$, then $U_{i}^{\prime} \cap B\left(x, \epsilon_{i}\right) \cap \operatorname{spt}\left(K_{i}\right)=$ $\emptyset$ for sufficiently large $i$ and both sides in (14) are zero. If $x \in \operatorname{spt}(\mu) \cap U^{\prime}$, then $B\left(x, \epsilon_{i}\right) \subset U_{i}^{\prime}$ for sufficiently large $i$ and both sides of (14) are equal to $\mu(x)$ by (10). Now let $x \in \operatorname{spt}(\mu) \cap l$. By enlarging the numbers $\epsilon_{i}$ if necessary, without changing the metrics $g_{i}$, we may assume that the geodesic line $l_{i}$ intersects $B\left(x, \epsilon_{i}\right)$ in a single arc for $i$ sufficiently large. Denote by $\sigma_{i}^{\prime} \in(0, \pi)$ the sum of the two exterior angles of $U_{i}^{\prime} \cap B\left(x, \epsilon_{i}\right)$ at the endpoints of this arc. Then

$$
\int_{U_{i}^{\prime} \cap B\left(x, \epsilon_{i}\right)} K_{i} d A_{i}+\int_{U_{i}^{\prime} \cap \partial B\left(x, \epsilon_{i}\right)} \kappa d s+\sigma_{i}^{\prime}=2 \pi .
$$

It is not difficult to see that the expression $\int_{U_{i}^{\prime} \cap \partial B\left(x, \epsilon_{i}\right)} \kappa d s+\sigma_{i}^{\prime}-\pi$ converges to the link $\lambda^{\prime}(x)$ of $P^{\prime}$ at $x$ as $i \rightarrow \infty$. Hence,

$$
\lim _{i \rightarrow \infty} \int_{U_{i}^{\prime} \cap B\left(x, \epsilon_{i}\right)} K_{i} d A_{i}=\pi-\lambda^{\prime}(x)=v_{P^{\prime}}(x) .
$$

This proves (14), and (15) follows analogously. In particular, using condition (ii) we conclude that $v_{P^{\prime}}(x), v_{P^{\prime \prime}}(x) \geq 0$ if $\mu(x) \geq 0$ and $v_{P^{\prime}}(x), v_{P^{\prime \prime}}(x) \leq 0$ if $\mu(x) \leq 0$. Thus, $l$ is a quasigeodesic line. By combining (14) and (15) with (12) we obtain

$$
\begin{aligned}
v_{P^{\prime}}^{+}\left(P^{\prime}\right) & =\lim _{i \rightarrow \infty} \int_{U_{i}^{\prime}} K_{i}^{+} d A_{i}=\mu^{+}(X) / 2, \\
v_{P^{\prime \prime}}^{+}\left(P^{\prime \prime}\right) & =\lim _{i \rightarrow \infty} \int_{U_{i}^{\prime \prime}} K_{i}^{+} d A_{i}=\mu^{+}(X) / 2 .
\end{aligned}
$$


Finally, we define

$$
\begin{aligned}
\eta^{\prime}(x) & :=\lim _{i \rightarrow \infty} \int_{U_{i}^{\prime} \cap B\left(x, \epsilon_{i}\right)} h_{i} d A_{i}, \\
\eta^{\prime \prime}(x) & :=\lim _{i \rightarrow \infty} \int_{U_{i}^{\prime \prime} \cap B\left(x, \epsilon_{i}\right)} h_{i} d A_{i}
\end{aligned}
$$

for all $x \in X$; then $\eta^{\prime}(x)+\eta^{\prime \prime}(x)=\lim _{i \rightarrow \infty} \int_{B\left(x, \epsilon_{i}\right)} h_{i} d A_{i}=\eta(x)$ by (11). In combination with (13) we get

$$
\begin{aligned}
\eta^{\prime}\left(P^{\prime}\right) & =\lim _{i \rightarrow \infty} \int_{U_{i}^{\prime}} h_{i} d A_{i}=\eta(X) / 2, \\
\eta^{\prime \prime}\left(P^{\prime \prime}\right) & =\lim _{i \rightarrow \infty} \int_{U_{i}^{\prime \prime}} h_{i} d A_{i}=\eta(X) / 2 .
\end{aligned}
$$

Note that for $x \in \operatorname{spt}(\mu) \cap \operatorname{spt}(\eta)$ we have

$$
\eta^{\prime}(x)=(\eta(x) / \mu(x)) \lim _{i \rightarrow \infty} \int_{U_{i}^{\prime} \cap B\left(x, \epsilon_{i}\right)} K_{i} d A_{i}=\eta(x) v_{P^{\prime}}(x) / \mu(x),
$$

and similarly $\eta^{\prime \prime}(x)=\eta(x) v_{P^{\prime \prime}}(x) / \mu(x)$.

By combining Propositions 5.2 and 6.2 we obtain an analog of Proposition 5.2 for polyhedral planes.

Proposition 6.3. Let $X$ be a polyhedral plane with only finitely many vertices and $\mu^{+}(X)<2 \pi$. Let $\alpha \geq 0$ be a measure on $X$ with finite support $\operatorname{spt}(\alpha)$ such that

$$
\alpha \geq \mu^{-} \text {and } 0<\alpha(X) \leq 2 \pi-\mu(X) .
$$

Then there exist sectors $\Sigma_{i}=\left\{(r \cos (\theta), r \sin (\theta)): r \geq 0,0 \leq \theta \leq \beta_{i}\right\}$ in $\mathbb{R}^{2}$ and arcwise isometric embeddings $\sigma_{i}: \Sigma_{i} \rightarrow X$, for $i=1,2, \ldots$ up to some finite index $k$, such that $\sigma_{i}(0) \in \operatorname{spt}(\alpha), \sigma_{i}\left(\operatorname{int}\left(\Sigma_{i}\right)\right) \cap \sigma_{j}\left(\operatorname{int}\left(\Sigma_{j}\right)\right)=\emptyset$ for $i \neq j$, and $\sum_{\sigma_{i}(0)=x} \beta_{i}=\alpha(x)$ for every $x \in \operatorname{spt}(\alpha)$.

Proof. We have $\eta:=\alpha+\mu=\alpha+\mu^{+}-\mu^{-} \geq \mu^{+} \geq 0$. According to Proposition 6.2 there exist a properly embedded, complete quasigeodesic line $l \subset X$ and measures $\eta^{\prime}, \eta^{\prime \prime} \geq 0$ on $X$ such that $\eta^{\prime}+\eta^{\prime \prime}=\eta$, $\operatorname{spt}\left(\eta^{\prime}\right) \subset P^{\prime}, \operatorname{spt}\left(\eta^{\prime \prime}\right) \subset P^{\prime \prime}$,

$$
\begin{aligned}
v_{P^{\prime}}^{+}\left(P^{\prime}\right)=v_{P^{\prime \prime}}^{+}\left(P^{\prime \prime}\right) & =\mu^{+}(X) / 2<\pi \\
\text { and } \quad \eta^{\prime}\left(P^{\prime}\right)=\eta^{\prime \prime}\left(P^{\prime \prime}\right) & =\eta(X) / 2,
\end{aligned}
$$

where $P^{\prime}, P^{\prime \prime}$ denote the closures of the connected components of $X \backslash l$ and $v_{P^{\prime}}, v_{P^{\prime \prime}}$ the corresponding total measures. Moreover, $\eta^{\prime}(x)=\eta(x) v_{P^{\prime}}(x) / \mu(x)$ and $\eta^{\prime \prime}(x)=\eta(x) v_{P^{\prime \prime}}(x) / \mu(x)$ for all $x \in \operatorname{spt}(\mu)$. Since $\eta \geq \mu^{+}$it follows that $\eta^{\prime} \geq v_{P^{\prime}}^{+}\left(\right.$note that $v_{P^{\prime}}(x)>0$ implies $\mu(x)>0$ ). Thus, $\alpha^{\prime}:=\eta^{\prime}-v_{P^{\prime}} \geq v_{P^{\prime}}^{-}$, and similarly $\alpha^{\prime \prime}:=\eta^{\prime \prime}-v_{P^{\prime \prime}} \geq v_{P^{\prime \prime}}^{-}$. We have

$$
\alpha^{\prime}\left(P^{\prime}\right)+v_{P^{\prime}}\left(P^{\prime}\right)=\eta^{\prime}\left(P^{\prime}\right)=\eta(X) / 2=(\alpha(X)+\mu(X)) / 2 \leq \pi
$$


by assumption, hence $\alpha^{\prime}\left(P^{\prime}\right) \leq \pi-v_{P^{\prime}}\left(P^{\prime}\right)$, and analogously $\alpha^{\prime \prime}\left(P^{\prime \prime}\right) \leq$ $\pi-v_{P^{\prime \prime}}\left(P^{\prime \prime}\right)$. (16) implies that the link of every interior vertex of $P^{\prime}$ or $P^{\prime \prime}$ is $>\pi$. The desired sectors and embeddings are now obtained by applying Proposition 5.2 to both $\left(P^{\prime}, \alpha^{\prime}\right)$ and $\left(P^{\prime \prime}, \alpha^{\prime \prime}\right)$ (provided $\alpha^{\prime} \neq 0$ or $\alpha^{\prime \prime} \neq 0$, respectively). The assertion $\sum_{\sigma_{i}(0)=x} \beta_{i}=\alpha(x)$ for every $x \in \operatorname{spt}(\alpha)$ follows since $\alpha^{\prime}+\alpha^{\prime \prime}=\eta^{\prime}+\eta^{\prime \prime}-v_{P^{\prime}}-v_{P^{\prime \prime}}=\eta-\mu=\alpha$.

\section{Bi-Lipschitz parameterization}

We first prove a bi-Lipschitz parameterization theorem for polyhedral halfplanes which does not use the results of Section 6.

Theorem 7.1. Let $(P, d)$ be a polyhedral halfplane with boundary $\partial P$ and only finitely many interior and boundary vertices. Denote by $v=\mu+\tau$ the total measure of $P$, and suppose that $v^{+}(P)<\pi$. Then there exists a homeomorphism $f: P \rightarrow\left\{(u, v) \in \mathbb{R}^{2}: v \geq 0\right\}$ satisfying

$$
\frac{\pi}{\pi+v^{-}(P)} d(x, y) \leq|f(x)-f(y)| \leq \frac{\pi}{\pi-v^{+}(P)} d(x, y)
$$

for all $x, y \in P$, with the property that $f \mid \partial P$ preserves arclength. In particular, $P$ is L-bi-Lipschitz equivalent to a closed Euclidean halfplane for

$$
L=\left(\frac{\pi+v^{-}(P)}{\pi-v^{+}(P)}\right)^{1 / 2} .
$$

Proof. The required bi-Lipschitz equivalence is constructed in two steps. In the first step we eliminate the positive part of $v$. More precisely, we define a new polyhedral metric $d_{1}$ on $P$, bi-Lipschitz equivalent to $d$, such that the corresponding total measure $v_{1}$ is non-positive. In the second step we eliminate the negative part of the curvature by constructing a polyhedral metric $d_{2}$ on $P$, bi-Lipschitz equivalent to $d_{1}$, such that $\left(P, d_{2}\right)$ is isometric to a closed Euclidean halfplane.

Step 1. Let

$$
L_{1}:=\frac{\pi}{\pi-v^{+}(P)} .
$$

If $v^{+}(P)=0$, put $d_{1}:=d$. Now suppose $v^{+}(P)>0$. Define a measure $\alpha_{1}$ on $P$ by

$$
\alpha_{1}:=\frac{\pi-v^{+}(P)}{v^{+}(P)} v^{+}+v^{-} .
$$

Then we have $\alpha_{1} \geq v^{-}$and

$$
0<\alpha_{1}(P)=\pi-v^{+}(P)+v^{-}(P)=\pi-v(P) .
$$

Since $v^{+}(P)<\pi$, the link of every interior vertex of $P$ is $>\pi$. Proposition 5.2 yields sectors $\Sigma_{i}=\left\{(r \cos (\theta), r \sin (\theta)): r \geq 0,0 \leq \theta \leq \beta_{i}\right\}$ in $\mathbb{R}^{2}$ and arcwise 
isometric embeddings $\sigma_{i}: \Sigma_{i} \rightarrow(P, d)$, for $i=1, \ldots, k$, such that $\sigma_{i}(0) \in$ $\operatorname{spt}\left(\alpha_{1}\right)=\operatorname{spt}(v), \sigma_{i}\left(\operatorname{int}\left(\Sigma_{i}\right)\right) \cap \sigma_{j}\left(\operatorname{int}\left(\Sigma_{j}\right)\right)=\emptyset$ for $i \neq j$, and $\Sigma_{\sigma_{i}(0)=x} \beta_{i}=$ $\alpha_{1}(x)$ for every $x \in \operatorname{spt}(v)$. Now $d_{1}$ is defined as the intrinsic metric on $P$ with the following properties: Let $c:[a, b] \rightarrow P$ be a $d$-rectifiable curve, i.e. $L(c)<\infty$, and denote by $L_{d_{1}}(c)$ its length with respect to $d_{1}$. If $\operatorname{im}(c) \cap \sigma_{i}\left(\operatorname{int}\left(\Sigma_{i}\right)\right)=\emptyset$ for all $i$ with $\sigma_{i}(0) \in \operatorname{spt}\left(v^{+}\right)$, then $L_{d_{1}}(c)=L(c)$. If $\operatorname{im}(c) \subset \sigma_{i}\left(\Sigma_{i}\right)$ for some $i$ with $\sigma_{i}(0) \in \operatorname{spt}\left(v^{+}\right)$, then $L_{d_{1}}(c)=L\left(\phi_{i} \circ \sigma_{i}^{-1} \circ c\right)$, where $\phi_{i}: \Sigma_{i} \rightarrow \mathbb{R}^{2}$ is defined by

$$
\phi_{i}(r \cos (\theta), r \sin (\theta))=\left(r \cos \left(L_{1} \theta\right), r \sin \left(L_{1} \theta\right)\right)
$$

for $r \geq 0,0 \leq \theta \leq \beta_{i}$. These properties determine, via subdivision, the $d_{1}$-length of every rectifiable curve in $P$ and, hence, $d_{1}$. Since $L_{1} \geq 1$ it follows that

$$
d(x, y) \leq d_{1}(x, y) \leq L_{1} d(x, y)
$$

for all $x, y \in P$. Moreover, $d_{1}$ is a complete, geodesic, polyhedral metric on $P$ whose vertex set is contained in the vertex set of $d$. We claim that the total measure $v_{1}$ of $\left(P, d_{1}\right)$ satisfies $v_{1}^{+}=0$ and $v_{1}^{-}=v^{-}$. The latter assertion is clear since $d_{1}$ agrees with $d$ in a neighborhood of every element of $\operatorname{spt}\left(v^{-}\right)$. If $x \in \operatorname{spt}\left(v^{+}\right)$, then the link of $x$ with respect to $d_{1}$ is

$$
\begin{aligned}
\lambda_{1}(x) & =\lambda(x)-\alpha_{1}(x)+L_{1} \alpha_{1}(x) \\
& =\lambda(x)+v^{+}(x) \\
& = \begin{cases}2 \pi & \text { if } x \in \operatorname{int}(P), \\
\pi & \text { if } x \in \partial P .\end{cases}
\end{aligned}
$$

This shows that $v_{1}(x)=0$.

Step 2. Let

$$
L_{2}:=\frac{\pi}{\pi+v^{-}(P)} .
$$

If $v^{-}(P)=0$, put $d_{2}:=d_{1}$. Now suppose $v^{-}(P)>0$. Define a measure $\alpha_{2}$ on $P$ by

$$
\alpha_{2}:=\frac{\pi+v^{-}(P)}{v^{-}(P)} v^{-} .
$$

Then we have $\alpha_{2} \geq v^{-}=v_{1}^{-}$and, since $v_{1}^{+}=0$,

$$
0<\alpha_{2}(P)=\pi+v^{-}(P)=\pi-v_{1}(P) .
$$

Proposition 5.2 yields sectors $\Sigma_{i}^{\prime}=\left\{(r \cos (\theta), r \sin (\theta)): r \geq 0,0 \leq \theta \leq \beta_{i}^{\prime}\right\}$ in $\mathbb{R}^{2}$ and arcwise isometric embeddings $\sigma_{i}^{\prime}: \Sigma_{i}^{\prime} \rightarrow\left(P, d_{1}\right)$, for $i=1, \ldots, k^{\prime}$, such that $\sigma_{i}^{\prime}(0) \in \operatorname{spt}\left(\alpha_{2}\right)=\operatorname{spt}\left(v_{1}\right), \sigma_{i}^{\prime}\left(\operatorname{int}\left(\Sigma_{i}^{\prime}\right)\right) \cap \sigma_{j}^{\prime}\left(\operatorname{int}\left(\Sigma_{j}^{\prime}\right)\right)=\emptyset$ for $i \neq j$, and $\Sigma_{\sigma_{i}^{\prime}(0)=x} \beta_{i}^{\prime}=\alpha_{2}(x)$ for every $x \in \operatorname{spt}\left(v_{1}\right)$. Now $d_{2}$ is defined as the intrinsic metric on $P$ determined by the following properties: Let $c:[a, b] \rightarrow P$ be a $d_{1}$-rectifiable curve. If $\operatorname{im}(c) \cap \sigma_{i}^{\prime}\left(\operatorname{int}\left(\Sigma_{i}^{\prime}\right)\right)=\emptyset$ for all $i$, then $L_{d_{2}}(c)=L_{d_{1}}(c)$. If 
$\operatorname{im}(c) \subset \sigma_{i}^{\prime}\left(\Sigma_{i}^{\prime}\right)$ for some $i$, then $L_{d_{2}}(c)=L_{d_{1}}\left(\phi_{i}^{\prime} \circ \sigma_{i}^{-1} \circ c\right)$, where $\phi_{i}^{\prime}: \Sigma_{i}^{\prime} \rightarrow \mathbb{R}^{2}$ is defined by

$$
\phi_{i}^{\prime}(r \cos (\theta), r \sin (\theta))=\left(r \cos \left(L_{2} \theta\right), r \sin \left(L_{2} \theta\right)\right)
$$

for $r \geq 0,0 \leq \theta \leq \beta_{i}^{\prime}$. Since $L_{2} \leq 1$ it follows that

$$
L_{2} d_{1}(x, y) \leq d_{2}(x, y) \leq d_{1}(x, y)
$$

for all $x, y \in P$. Moreover, $d_{2}$ is a complete, geodesic, polyhedral metric on $P$ whose vertex set is contained in the vertex set of $d_{1}$. We claim that $\left(P, d_{2}\right)$ is isometric to a closed Euclidean halfplane. If $x \in \operatorname{spt}\left(v_{1}\right)$, then the link of $x$ with respect to $d_{2}$ is

$$
\begin{aligned}
\lambda_{2}(x) & =\lambda(x)-\alpha_{2}(x)+L_{2} \alpha_{2}(x) \\
& =\lambda(x)-v^{-}(x) \\
& = \begin{cases}2 \pi & \text { if } x \in \operatorname{int}(P), \\
\pi & \text { if } x \in \partial P .\end{cases}
\end{aligned}
$$

This proves the claim. By combining (17) with (18) and identifying $\left(P, d_{2}\right)$ with $\left\{(u, v) \in \mathbb{R}^{2}: v \geq 0\right\}$ we get the desired map $f$ satisfying

$$
L_{2} d(x, y) \leq|f(x)-f(y)| \leq L_{1} d(x, y)
$$

for all $x, y \in P$. From the construction it is clear that $f \mid \partial P$ preserves arclength. By rescaling $f$ we obtain an $L$-bi-Lipschitz equivalence of $P$ with a closed Euclidean halfplane for $L=\left(L_{1} / L_{2}\right)^{1 / 2}$.

Now we prove the polyhedral version of our main result.

Theorem 7.2. Let $X$ be a polyhedral plane with only finitely many vertices and $\mu^{+}(X)<2 \pi$. Then $X$ is L-bi-Lipschitz equivalent to the Euclidean plane for

$$
L=\left(\frac{2 \pi+\mu^{-}(X)}{2 \pi-\mu^{+}(X)}\right)^{1 / 2} .
$$

Proof. We use Proposition 6.2 to find a properly embedded, complete quasigeodesic line $l$ in $X$ such that

$$
\text { and } \begin{aligned}
v_{P^{\prime}}^{+}\left(P^{\prime}\right) & =v_{P^{\prime \prime}}^{+}\left(P^{\prime \prime}\right)=\mu^{+}(X) / 2<\pi \\
v_{P^{\prime}}^{-}\left(P^{\prime}\right) & =v_{P^{\prime \prime}}^{-}\left(P^{\prime \prime}\right)=\mu^{-}(X) / 2,
\end{aligned}
$$

where $P^{\prime}, P^{\prime \prime}$ denote the closures of the connected components of $X \backslash l$, endowed with their induced intrinsic metrics, and $v_{P^{\prime}}, v_{P^{\prime \prime}}$ denote the corresponding total measures. Applying Theorem 7.1 twice we produce a homeomorphism $f: X \rightarrow$ 
$\mathbb{R}^{2}$ such that $f \mid l$ preserves arclength and both $f \mid P^{\prime}: P^{\prime} \rightarrow\left\{(u, v) \in \mathbb{R}^{2}: v \geq 0\right\}$ and $f \mid P^{\prime \prime}: P^{\prime \prime} \rightarrow\left\{(u, v) \in \mathbb{R}^{2}: v \leq 0\right\}$ satisfy

$$
L_{2} d(x, y) \leq|f(x)-f(y)| \leq L_{1} d(x, y)
$$

for all $x, y$ in the respective domain, where

$$
\begin{aligned}
& L_{1}=\frac{\pi}{\pi-v_{P^{\prime}}^{+}\left(P^{\prime}\right)}=\frac{\pi}{\pi-v_{P^{\prime \prime}}^{+}\left(P^{\prime \prime}\right)}=\frac{2 \pi}{2 \pi-\mu^{+}(X)}, \\
& L_{2}=\frac{\pi}{\pi+v_{P^{\prime}}^{-}\left(P^{\prime}\right)}=\frac{\pi}{\pi+v_{P^{\prime \prime}}^{-}\left(P^{\prime \prime}\right)}=\frac{2 \pi}{2 \pi+\mu^{-}(X)} .
\end{aligned}
$$

It follows easily that $f$ satisfies (19) for all $x, y \in X$. By rescaling $f$ we obtain the desired $L$-bi-Lipschitz equivalence of $X$ with $\mathbb{R}^{2}$ for $L=\left(L_{1} / L_{2}\right)^{1 / 2}$.

Another way to prove the theorem is to put

$$
\alpha_{1}:=\frac{2 \pi-\mu^{+}(X)}{\mu^{+}(X)} \mu^{+}+\mu^{-}, \quad \alpha_{2}:=\frac{2 \pi+\mu^{-}(X)}{\mu^{-}(X)} \mu^{-}
$$

(provided $\mu^{+}(X)>0$ or $\mu^{-}(X)>0$, respectively), and to proceed as in the proof of Theorem 7.1, using Proposition 6.3 instead of Proposition 5.2.

\section{Proof of Theorem 1.1}

In this section we will deduce Theorem 1.1 from Theorem 7.2 by an approximation argument. Aleksandrov surfaces are exactly those surfaces with intrinsic metric that allow approximations by polyhedral surfaces in an appropriate local sense (see [AZ, Chapter 4, Section 1] for more discussion). Moreover, these approximations can be arranged so that the positive and negative parts of the integral curvatures of the approximating polyhedral surfaces converge locally weakly to the positive and negative parts of the integral curvature of the surface being approximated ([AZ, p. 246, Theorem 7]). In view of this it seems "obvious" that the general case of Theorem 1.1 follows from the case of polyhedral surfaces. A closer look reveals that there are considerable subtleties that have to be addressed. Since the weak convergence of the curvatures of an approximating sequence of polyhedral surfaces is only local, one has to work in a fixed compact part $P$ of the given surface $Z$. In this way, one incurs additional boundary terms in the curvature estimates (see inequalities (i) of Lemma 8.1). Moreover, on the compact part $P$ the polyhedral metrics approximate only the intrinsic metric $d_{P}$ on $P$ which will be different from the restriction of the ambient metric $d_{Z}$ to $P$. These problems necessitate a careful choice of the compact sets $P$ on which we approximate $Z$ by polyhedral surfaces. The intrinsic metric $d_{P}$ will agree with the restriction of $d_{Z}$ to $P$ if $P$ is convex. Moreover, the boundary terms in the curvature estimates will be manageable if $P$ is a Jordan polygon which is convex with respect to its 
boundary. This is the reason why we proved Lemma 3.3 which provides a suitable exhaustion of a surface $Z$ satisfying the hypotheses of Theorem 1.1.

We now proceed to the details of the approximation argument.

Lemma 8.1. Suppose $Z$ is a complete Aleksandrov surface homeomorphic to the plane, $P \subset Z$ a convex Jordan polygon, and $\epsilon>0$. Then there exist a polyhedral disk $P^{\prime}$ and a homeomorphism $f: P \rightarrow P^{\prime}$ such that

(i) $\mu_{P^{\prime}}^{+}\left(\operatorname{int}\left(P^{\prime}\right)\right) \leq \mu_{Z}^{+}(\operatorname{int}(P))$ and

$$
\mu_{P^{\prime}}^{-}\left(\operatorname{int}\left(P^{\prime}\right)\right)+\tau^{-}\left(\partial P^{\prime}\right) \leq \mu_{Z}^{-}(\operatorname{int}(P))+\tau_{\text {in }}^{-}(\partial P),
$$

(ii) $d_{Z}(x, y)-\epsilon \leq d_{P^{\prime}}(f(x), f(y)) \leq d_{Z}(x, y)+\epsilon \quad$ for $x, y \in P$.

Proof. We consider a triangulation $T$ of $P$ by simple triangles (as defined in Section 2) with small diameter. For general Aleksandrov surfaces, the term triangulation has to be understood in a certain non-standard sense (see [AZ, p. 61, Theorem 3] for a precise existence statement). From $T$ we obtain a polyhedral surface $P^{\prime}$ as follows. We replace each triangle in the triangulation $T$ by a Euclidean triangle with equal side lengths and glue the Euclidean triangles together according to the combinatorial pattern of the triangulation $T$. In the gluing we respect arclength on the parts of triangle edges which are identified. In this way we also obtain a natural map $f$ from the 1-skeleton of the triangulation $T$ of $P$ to the 1-skeleton of the induced triangulation $T^{\prime}$ of $P^{\prime}$. The map $f$ can be extended to a homeomorphism $f: P \rightarrow P^{\prime}$ by mapping each triangle in $T$ homeomorphically to the corresponding triangle in $T^{\prime}$ in an arbitrary way under the restriction that we respect the definition of $f$ on the boundary of the triangle (see [AZ, Chapter III, Sections 4 and 6]).

Since $P$ is convex, the intrinsic metric $d_{P}$ agrees with the restriction of the ambient metric $d$ to $P$. This implies that if the maximal diameter of the triangles in $T$ is small enough, then (ii) will be valid (see [AZ, Chapter III, Section 6, and in particular p. 79, Theorem 9 and p. 84, Theorem 10]).

The inequalities in (i) follow from the fact that a polyhedral approximation as in the first part of the proof reduces both the positive and negative part of the curvature if one takes boundary terms into proper account (see [AZ, p. 245, Lemma 19; note the typographical error in the first inequality (35)]).

Lemma 8.2. Suppose $Z$ is a complete Aleksandrov surface homeomorphic to the plane and $P \subset Z$ a Jordan polygon which is convex relative to its boundary. Then for all $\epsilon>0$ there exist a polyhedral plane $Z^{\prime}$ with finitely many vertices, a Jordan polygon $P^{\prime} \subset Z^{\prime}$ which is convex relative to its boundary and a homeomorphism $f: P \rightarrow P^{\prime}$ such that

(i) $\mu_{Z^{\prime}}^{+}\left(Z^{\prime}\right) \leq \mu_{Z}^{+}(Z)$ and $\mu_{Z^{\prime}}^{-}\left(Z^{\prime}\right) \leq \mu_{Z}^{-}(Z)$,

(ii) $d_{Z}(x, y)-\epsilon \leq d_{Z^{\prime}}(f(x), f(y)) \leq d_{Z}(x, y)+\epsilon$ for all $x, y \in P$. 
Note in particular that if $Z$ is non-negatively or non-positively curved, then $Z^{\prime}$ enjoys the same property.

Proof. Let $P^{\prime}$ be as in the previous lemma. Then (ii) holds for $d_{P^{\prime}}$ in place of $d_{Z^{\prime}}$.

Along the boundary of $P^{\prime}$ we glue a polyhedral surface $P^{\prime \prime}$ homeomorphic to the complement of an open disk so that $Z^{\prime}=P^{\prime} \cup P^{\prime \prime}$ is a complete polyhedral surface homeomorphic to the plane with finitely many vertices, and $P^{\prime} \subset Z^{\prime}$ is convex relative to its boundary (in the following all gluings will respect arclength). To do this decompose $\partial P^{\prime}$ into non-overlapping shortest subarcs $\gamma_{1}, \ldots, \gamma_{n}$ arranged in cyclic order, and denote by $v_{1}, \ldots, v_{n}=v_{0}$ the vertices of $\partial P^{\prime}$ in cyclic order so that $\gamma_{i}$ has the endpoints $v_{i-1}$ and $v_{i}$. Let $s_{i}$ be the length of $\gamma_{i}$ and $\alpha_{i}$ be the angle of the interior sector at $v_{i}$. Now define

$$
\beta_{i}:= \begin{cases}2 \pi-\alpha_{i} & \text { for } 0 \leq \alpha_{i} \leq \pi, \\ \pi & \text { for } \alpha_{i} \geq \pi,\end{cases}
$$

and $\beta_{i}^{\prime}:=\beta_{i}-\pi \geq 0$. Consider half-strips $Q_{i}:=\left\{(x, y) \in \mathbb{R}^{2} 0 \leq x \leq s_{i}\right.$, $y \geq 0\}$ of width $s_{i}$ and (possibly degenerate) sectors $\Sigma_{i}:=\{(r \cos (\theta), r \sin (\theta))$ : $\left.r \geq 0,0 \leq \theta \leq \beta_{i}^{\prime}\right\}$ of opening angle $\beta_{i}^{\prime}$. Glue them together in cyclic order $Q_{1}, \Sigma_{1}$, $Q_{2}, \ldots, Q_{n}, \Sigma_{n}, Q_{n+1}=Q_{1}$ such that the ray $\left\{\left(s_{i}, y\right): y \geq 0\right\}$ in $Q_{i}$ is identified with the ray $\{(r, 0): r \geq 0\}$ in $\Sigma_{i}$, and the ray $\left\{\left(r \cos \left(\beta_{i}^{\prime}\right), r \sin \left(\beta_{i}^{\prime}\right)\right): r \geq 0\right\}$ in $\Sigma_{i}$ is identified with the ray $\{(0, y): y \geq 0\}$ in $Q_{i+1}$. In this way we obtain the polyhedral surface $P^{\prime \prime}$. The boundary $\partial P^{\prime \prime}$ consists of a Jordan curve which is formed by geodesic segments corresponding to the segments $\left[0, s_{i}\right] \subset Q_{i}$. We identify $\left[0, s_{i}\right]$ with $\alpha_{i}$ so that $0 \in\left[0, s_{i}\right]$ corresponds to $v_{i-1}$ and $s_{i} \in\left[0, s_{i}\right]$ to $v_{i}$.

As desired, the surface $Z^{\prime}$ obtained from the gluing is complete and homeomorphic to the plane. Note that the angle at $v_{i}$ as seen from $P^{\prime \prime}$ is equal to $\beta_{i}$ and hence at least $\pi$ by the definition of $\beta_{i}$. This implies that for any rectifiable curve in $P^{\prime \prime}$ with endpoints in $\partial P^{\prime \prime}=\partial P^{\prime}$ which meets the interior of $P^{\prime \prime}$ we can find a homotopic curve in $\partial P^{\prime \prime}$ with the same endpoints which has strictly smaller length. This shows that $P^{\prime}$ is convex relative to its boundary and in particular convex. Hence $d_{P^{\prime}}$ is equal to the restriction of the ambient metric $d_{Z^{\prime}}$ to $P^{\prime}$, and so (ii) is true by our choice of $P^{\prime}$.

Every vertex of $Z^{\prime}$ is contained $\operatorname{in} \operatorname{int}\left(P^{\prime}\right)$ or equal to one of the vertices $v_{i}$. For the total angle at $v_{i}$ we have

$$
\lambda\left(v_{i}\right)=\alpha_{i}+\beta_{i} \geq 2 \pi
$$

and so $\mu_{Z^{\prime}}^{+}\left(v_{i}\right)=0$. Hence by the choice of $P^{\prime}$ and by the first inequality in (i) of Lemma 8.1 we get

$$
\mu_{Z^{\prime}}^{+}\left(Z^{\prime}\right)=\mu_{P^{\prime}}^{+}\left(\operatorname{int}\left(P^{\prime}\right)\right) \leq \mu_{Z}^{+}(\operatorname{int}(P)) \leq \mu_{Z}^{+}(Z) .
$$

This shows the first inequality in (i). 
For the second inequality in (i) note that for the turn $\tau\left(v_{i}\right)$ of $\partial P^{\prime}$ at $v_{i}$ (as seen from inside $\left.P^{\prime}\right)$ we have $\tau\left(v_{i}\right)=\pi-\alpha_{i}$. Since $\mu_{Z^{\prime}}\left(v_{i}\right)=2 \pi-\alpha_{i}-\beta_{i}$, it follows from the definition of $\beta_{i}$ that $\mu_{Z^{\prime}}^{-}\left(v_{i}\right)=\tau^{-}\left(v_{i}\right)$. Hence by the second inequality in (i) of Lemma 8.1 we get

$$
\mu_{Z^{\prime}}^{-}\left(Z^{\prime}\right)=\mu_{P^{\prime}}^{-}\left(\operatorname{int}\left(P^{\prime}\right)\right)+\tau^{-}\left(\partial P^{\prime}\right) \leq \mu_{Z}^{-}(\operatorname{int}(P))+\tau_{\text {in }}^{-}(\partial P) .
$$

Let $\tau_{\text {out }}$ be the turn on $\partial P$ as seen from outside $P$ considered as Borel measure on $\partial P$. Since $P$ is convex relative to its boundary, we have $\tau_{\text {out }} \leq 0$. Let $\tau_{\text {in }}=\tau_{\text {in }}^{+}-\tau_{\text {in }}^{-}$ and $\tau_{\text {out }}=\tau_{\text {out }}^{+}-\tau_{\text {out }}^{-}$be the Jordan decompositions of $\tau_{\text {in }}$ and $\tau_{\text {out }}$, respectively. Then $\tau_{\text {out }}^{+}=0$. Moreover, if $E \subset \partial P$ is a Borel set, then

$$
\tau_{\text {in }}(E)+\tau_{\text {out }}(E)=\mu_{Z}(E)
$$

and so

$$
\tau_{\text {in }}^{+}(E)-\tau_{\text {in }}^{-}(E)=\tau_{\text {out }}^{-}(E)+\mu_{Z}^{+}(E)-\mu_{Z}^{-}(E) .
$$

Thus, by the minimality property of the Jordan decomposition we get

$$
\tau_{\text {in }}^{-}(E) \leq \mu_{Z}^{-}(E)
$$

for every Borel set $E \subset \partial P$. This inequality for $E=\partial P$ together with (20) implies the second inequality in (i).

We now prove Theorem 1.1 which we restate as follows.

Theorem 8.3. Suppose $Z$ is a complete Aleksandrov surface homeomorphic to the plane. If $\mu^{+}(Z)<2 \pi$ and $\mu^{-}(Z)<\infty$, then $Z$ is L-bi-Lipschitz equivalent to $\mathbb{R}^{2}$ with

$$
L=\left(\frac{2 \pi+\mu^{-}(Z)}{2 \pi-\mu^{+}(Z)}\right)^{1 / 2} \text {. }
$$

Proof. Let $Z$ be a surface satisfying our hypotheses, and let $L \geq 1$ be the constant as defined in the statement of the theorem. By Lemma 3.3 we can find Jordan polygons $P_{n}$ in $Z$ for $n \in \mathbb{N}$ which are convex relative to their boundaries such that $P_{n} \subset \operatorname{int}\left(P_{n+1}\right)$ and $\bigcup_{n \in \mathbb{N}} P_{n}=Z$. According to Lemma 8.2 we can find polyhedral planes $Z_{n}^{\prime}$ with finitely many vertices, Jordan polygons $P_{n}^{\prime} \subset Z_{n}^{\prime}$ which are convex relative to their boundaries, and homeomorphisms $f_{n}: P_{n} \rightarrow P_{n}^{\prime}$ such that

$$
\mu_{Z_{n}^{\prime}}^{+}\left(Z_{n}^{\prime}\right) \leq \mu_{Z}^{+}(Z) \quad \text { and } \quad \mu_{Z_{n}^{\prime}}^{-}\left(Z_{n}^{\prime}\right) \leq \mu_{Z}^{-}(Z) \quad \text { for } n \in \mathbb{N}
$$

and

$$
d_{Z}(x, y)-1 / n \leq d_{Z_{n}^{\prime}}\left(f_{n}(x), f_{n}(y)\right) \leq d_{Z}(x, y)+1 / n
$$

for all $x, y \in P_{n}$ and $n \in \mathbb{N}$. By Theorem 7.2 we can find $L$-bi-Lipschitz homeomorphisms $g_{n}: Z_{n}^{\prime} \rightarrow \mathbb{R}^{2}$. Let $p_{0} \in P_{1}$ be a base point in $Z$. By composing $g_{n}$ 
with a translation of $\mathbb{R}^{2}$ is necessary, we can assume that $g_{n}\left(f_{n}\left(p_{0}\right)\right)=0$. Define $h_{n}:=g_{n} \circ f_{n}$. Then $h_{n}: P_{n} \rightarrow \mathbb{R}^{2}$ is a map satisfying $h_{n}\left(p_{0}\right)=0$ and

$$
\frac{1}{L} d_{Z}(x, y)-\frac{1}{n L} \leq\left|h_{n}(x)-h_{n}(y)\right| \leq \operatorname{Ld}_{Z}(x, y)+\frac{L}{n}
$$

for $x, y \in P_{n}, n \in \mathbb{N}$. Fix a countable dense subset $D \subset Z$. If $x \in D$, then $x \in P_{n}$ for sufficiently large $n$ and so $h_{n}(x)$ is defined. Moreover, due to (21) and the normalization, the points $h_{n}(x), n$ sufficiently large, form a bounded set in $\mathbb{R}^{n}$. By passing to successive subsequences of $\left(h_{n}\right)$ and choosing a final "diagonal" subsequence, it follows that we may assume that the sequence $\left(h_{n}(x)\right)$ converges for each $x \in D$. Define $h: D \rightarrow \mathbb{R}^{2}$ by setting $h(x):=\lim _{n \rightarrow \infty} h_{n}(x)$ for $x \in D$. Passing to limits in (21) we conclude that

$$
\frac{1}{L} d_{Z}(x, y) \leq|h(x)-h(y)| \leq L d_{Z}(x, y)
$$

for $x, y \in D$. This implies that $h$ has a unique extension to a map $h: Z \rightarrow \mathbb{R}^{2}$ satisfying (22) for all $x, y \in Z$. In particular, $h$ is an $L$-bi-Lipschitz embedding of $Z$ into $\mathbb{R}^{2}$. By the open mapping theorem, the image $h(Z)$ is open. Moreover, the set $h(Z)$ is also closed, as the image of a complete space under a bi-Lipschitz embedding. Thus, $h(Z)=\mathbb{R}^{2}$ and $h$ is the desired $L$-bi-Lipschitz homeomorphism of $Z$ onto $\mathbb{R}^{2}$.

\section{Concluding remarks}

(I) It is of independent interest to give a purely analytic version of our main result. By a uniformization theorem due to Reshetnyak and A. Huber, every orientable Aleksandrov surface $Z$ carries a unique conformal structure. If $Z$ is homeomorphic to the plane, then $Z$ is conformally equivalent to the plane or an open disk. According to these two possibilities one says that $Z$ has parabolic or hyperbolic type. If $|\mu|(Z)<\infty$ as for the surfaces in Theorem 8.3, then $Z$ is always of parabolic type. In this case there exists a global parametrization of the surface by a complex coordinate $z$ ranging in the complex plane $\mathbb{C}$ so that the length element has the representation as in (1).

More precisely, if $Z$ is an Aleksandrov surface homeomorphic to the plane with $|\mu|(Z)<\infty$, then it is isometric to the complex plane $\mathbb{C}$ equipped with the metric defined by

$$
d(x, y):=\inf _{\alpha} \int_{\alpha} \exp (u(z))|d z| \text { for } x, y \in \mathbb{C},
$$

where the infimum is taken over all analytic curves in $\mathbb{C}$ joining $x$ and $y$, and $u$ is the difference of two subharmonic functions on $\mathbb{C}$ such that every atom of the measure $-\Delta u$ has mass less than $2 \pi$. As we already remarked, the measure 
$\mu=-\Delta u$ corresponds to the integral curvature of the surface. In particular, if $|\mu|(\mathbb{C})<\infty$, then the function $u$ has the representation

$$
u(z)=h(z)+\frac{1}{2 \pi} \int_{\mathbb{C}} \log \left|\frac{z_{0}-\zeta}{z-\zeta}\right| d \mu(\zeta) \quad \text { for } z \in \mathbb{C},
$$

where $h$ is a harmonic function in the plane and $z_{0} \in \mathbb{C}$ is an appropriately chosen point. This choice has to be made so that the integral converges. This is true if the Hardy-Littlewood maximal function of $\mu$ is finite for $z_{0}$ (which holds for almost every point).

According to a result by A. Huber [Hu2], the function $h$ reduces to a constant if the surface is complete in addition. Then $Z$ is isometric to $(\mathbb{C}, d)$, where the metric $d$ is defined as in (23) for a function $u$ that has the representation

$$
u(z)=c+\frac{1}{2 \pi} \int_{\mathbb{C}} \log \left|\frac{z_{0}-\zeta}{z-\zeta}\right| d \mu(\zeta) .
$$

Here $c \in \mathbb{R}$ is a constant, and $\mu$ is a signed measure on $\mathbb{C}$ with finite total variation such that all atoms of $\mu$ have mass less than $2 \pi$.

Conversely, if we define the metric $d$ as in (23) by using a function $u$ as in (24), then $(\mathbb{C}, d)$ is a complete Aleksandrov surface. So our main theorem can be stated as follows: Suppose $\mu$ is a signed measure on $\mathbb{C}$ with $\mu^{+}(\mathbb{C})<2 \pi$ and $\mu^{-}(\mathbb{C})<\infty$. If we define $u$ as in (24) and the metric $d$ as in (23), then $(\mathbb{C}, d)$ is L-bi-Lipschitz equivalent to $\mathbb{C}$ with

$$
L=\left(\frac{2 \pi+\mu^{-}(\mathbb{C})}{2 \pi-\mu^{+}(\mathbb{C})}\right)^{1 / 2} .
$$

(II) The so-called Jacobian problem for quasiconformal homeomorphisms $f$ : $\mathbb{R}^{n} \rightarrow \mathbb{R}^{n}$ asks for a characterization of their Jacobian determinants $J_{f}$ up to multiplicative constants (see [Se2,BHS]). It can be shown that if $\rho$ is a reasonable conformal density on $\mathbb{C}$ such that $\mathbb{C}$ equipped with the metric coming from the length element $\rho(z)|d z|$ is $L$-bi-Lipschitz to $\mathbb{C}$, then there exists a $K$-quasiconformal map $f: \mathbb{C} \rightarrow \mathbb{C}$ and a constant $C \geq 1$ such that

$$
\frac{1}{C} J_{f}(z) \leq \rho(z)^{2} \leq C J_{f}(z) \text { for a.e. } z \in \mathbb{R}^{2} .
$$

The constants $K$ and $C$ will depend only on $L$. We can apply this fact in combination with our main theorem as formulated in the previous remark. To avoid technicalities we restrict ourselves to measures $\mu$ as in (24) which are supported in finitely many points (this corresponds to the case of a polyhedral surface $(Z, d)$ ), and obtain the following result: 
Theorem 9.1. Suppose $z_{1}, \ldots, z_{n}, w_{1}, \ldots, w_{m}$ are distinct points in $\mathbb{C}$, and suppose $\alpha_{1}, \ldots, \alpha_{n}, \beta_{1}, \ldots, \beta_{m}>0$ are positive numbers with $\alpha:=\alpha_{1}+\cdots+\alpha_{n}<$ 2. Set $\beta:=\beta_{1}+\cdots+\beta_{m}$. Then there exist constants $K, C \geq 1$ depending only on $\alpha$ and $\beta$ and a $K$-quasiconformal homeomorphism $f: \mathbb{C} \rightarrow \mathbb{C}$ such that

$$
\frac{1}{C} J_{f}(z) \leq \frac{\prod_{i=1}^{m}\left|z-w_{i}\right|^{\beta_{i}}}{\prod_{j=1}^{n}\left|z-z_{j}\right|^{\alpha_{j}}} \leq C J_{f}(z)
$$

for a.e. $z \in \mathbb{C}$.

By considering maps that look like radial stretchings near the points $w_{i}$ and $z_{j}$ it is not hard to construct a $K$-quasiconformal homeomorphism $f: \mathbb{C} \rightarrow \mathbb{C}$ satisfying (25) with some constants $K, C \geq 1$. The point of Theorem 9.1 is that $f$ can be chosen so that $K$ and $C$ depend only $\alpha$ and $\beta$. It would be very interesting to obtain a purely analytic proof of Theorem 9.1 by studying the Beltrami equation for example.

\section{References}

[AZ] Aleksandrov, A.D., Zalgaller, V.A.: Intrinsic Geometry of Surfaces. AMS Transl. Math. Monographs, Vol. 15, Providence, RI, 1967

[Ba1] Bangert, V.: Geodesics and totally convex sets on surfaces. Invent. Math. 63, 507-517 (1981)

[Ba2] Bangert, V.: On the existence of escaping geodesics. Comment. Math. Helvetici 56, 59-65 (1981)

[BE] Bonk, M., Eremenko, A.: Uniformly hyperbolic surfaces. Indiana Univ. Math. J. 49, 61-80 (2000)

[BHS] Bonk, M., Heinonen, J., Saksman, E.: The quasiconformal Jacobian problem. Preprint

[CV] Cohn-Vossen, St.: Kürzeste Wege und Totalkrümmung auf Flächen. Compositio Math. 2, 69-133 (1935)

[Fu] Fu, J.H.G.: Bi-Lipschitz rough normal coordinates for surfaces with an $L^{1}$ curvature bound. Indiana Univ. Math. J. 47, 439-453 (1998)

[GP] Gullemin, V., Pollack, A.: Differential Topology. Prentice-Hall, Englewood Cliffs, N.J., 1974

[Hu1] Huber, A.: Zum potentialtheoretischen Aspekt der Alexandrowschen Flächentheorie. Comment. Math. Helv. 34, 99-126 (1960)

[Hu2] Huber, A.: Vollständige konforme Metriken und isolierte Singularitäten subharmonischer Funktionen. Comment. Math. Helv. 41, 105-136 (1960)

[Mi] Milnor, J.: A problem in cartography. Amer. Math. Monthly 76, 1101-1112 (1969)

[MS] Müller, S., Šverák, V.: On surfaces of finite total curvature. J. Differential Geom. 42, 229-258 (1995)

[Re] Reshetnyak, Y.G.: Two-dimensional manifolds of bounded curvature. pp. 3-163. In: Y.G. Reshetnyak (Ed.), Geometry IV, Encyclopaedia of Math. Sci., Vol. 70, Springer 1993

[Se1] Semmes, S.: Hypersurfaces in $\mathbb{R}^{n}$ whose normal has small BMO norm. Proc. Amer. Math. Soc. 112, 403-412 (1991)

[Se2] Semmes, S.: Bi-Lipschitz mappings and strong $A_{\infty}$ weights. Ann. Acad. Sci. Fenn. Ser. A I Math. 18, 211-248 (1993) 
[Se3] Semmes, S.: On the nonexistence of bilipschitz parameterizations and geometric problems about $A_{\infty}$-weights. Rev. Mat. Iberoamer. 12, 337-410 (1996)

[To1] Toro, T.: Surfaces with generalized second fundamental form in $L^{2}$ are Lipschitz manifolds. J. Differential Geom. 39, 65-101 (1994)

[To2] Toro, T.: Geometric conditions and existence of bi-Lipschitz parameterizations. Duke Math. J. 77, 193-227 (1995) 